TRANSACTIONS OF THE

AMERICAN MATHEMATICAL SOCIETY

Volume 351, Number 3, March 1999, Pages 1123-1150

S $0002-9947(99) 02405-8$

\title{
A GENERALIZATION OF SNAITH-TYPE FILTRATION
}

\author{
GREG ARONE
}

\begin{abstract}
In this paper we describe the Goodwillie tower of the stable homotopy of a space of maps from a finite-dimensional complex to a highly enough connected space. One way to view it is as a partial generalization of some wellknown results on stable splittings of mapping spaces in terms of configuration spaces.
\end{abstract}

\section{INTRODUCTION}

It has been known for a while (see [1] for a survey article and a list of references) that given a parallelizable, compact $m$-dimensional manifold $M$ with a nonempty boundary, and given a connected, pointed space $Z$, there is a configuration space model for the space of unbased maps $\operatorname{Map}\left(M, S^{m} Z\right)$, which stably splits. More precisely, there is a weak equivalence:

$$
\Omega^{\infty} \Sigma^{\infty}\left(\operatorname{Map}\left(M, S^{m} Z\right)\right) \simeq \prod_{n \geq 1} \Omega^{\infty} \Sigma^{\infty}\left(\left(C(M, \partial M ; n) \wedge_{\Sigma_{n}} Z^{\wedge n}\right)\right),
$$

where $C(M, \partial M ; n)$ stands for the space of $n$-tuples of distinct points in $M$, where all $n$-tuples whose intersection with $\partial M$ is not empty have been identified to a point. There is an analogous splitting for the space of based maps. A closely related result is the stable splitting of spaces of the form $\Omega^{m} \Sigma^{m} X$. This later splitting is sometimes refered to as the Snaith splitting (at least in the case $m=\infty$ ), and we refer to (0.1) as Snaith-type splitting.

It is, therefore, natural to ask if for a based space $K$, that is not a manifold, but, say, a finite CW-complex, anything can be said about the functor $X \mapsto Q \operatorname{Map}_{*}(K, X)$ (where $\operatorname{Map}_{*}(K, X)$ stands for the space of based maps from $K$ to $X)$. One does not expect this functor to split, in general, but it is still reasonable to try to approximate it by more elementary functors in a way that would give the splitting above in the case when $K=M$ and $X=S^{m} Z$. It turns out that this question (in fact a generalization of it) can be answered positively within the framework of the theory referred to as calculus of functors, which had been developed by T. Goodwillie in [4], [5], [6]. Since [6] has not been published yet, we present a brief outline of the theory of "Taylor towers" in the appendix. In what follows we will freely use notation from there.

Consider again the identity (0.1). Observe that the factors

$$
Q\left(C(M, \partial M ; n) \wedge_{\Sigma_{n}} Z^{\wedge n}\right)
$$

Received by the editors July 21, 1994 and, in revised form, February 4, 1997. 1991 Mathematics Subject Classification. Primary 55P99.

(C)1999 American Mathematical Society 
are homogeneous functors (of $Z$ ). Therefore, the functors

$$
P_{n} \operatorname{Map}\left(M, S^{m} Z\right)=\prod_{i=0}^{i=n} Q\left(C(M, \partial M ; i) \wedge_{\Sigma_{i}} Z^{\wedge i}\right)
$$

are excisive of degree $n$. Moreover, it is, in fact, true that the (weak) map

$$
Q \operatorname{Map}\left(M, S^{m} Z\right) \rightarrow P_{n} \operatorname{Map}\left(M, S^{m} Z\right)
$$

is $(n+1) k$-connected if the space $Z$ is $k$-connected. Therefore, by universality, the right hand side of $(0.1)$ is nothing but the Taylor tower of the functor $Z \mapsto$ $Q \operatorname{Map}\left(M, S^{m} Z\right)$, where $Z \in T_{*}$. One can think of it as the "Maclaurin tower" of $Q \operatorname{Map}\left(M, S^{m} Z\right)$. Hence the generalization that we seek in this paper is the one of identifying the Taylor tower of the functor $Q \operatorname{Map}_{*}(K, Y)$, where $K$ is a finite CWcomplex and $Y \in T_{X}$. More concretely, we want to answer the following question: given a space $Y$ containing $X$ as a retract, how can we describe $Q \operatorname{Map}_{*}(K, Y)$ as a sequence of extensions of $Q \operatorname{Map}_{*}(K, X)$ by homogeneous functors?

Let us discuss the case $X=*$ (Maclaurin tower) first. We use implicitly the fact that the differentials of a functor can be recovered from the differentials of its composition with ( $m$-fold) suspension. Indeed, there are equivalences (the second of which follows essentially from duality on manifolds, although in our proofs we do not refer to duality directly)

$$
\begin{aligned}
D_{n}\left(Q \operatorname{Map}\left(M, S^{m} Z\right)\right) & \simeq Q\left(C(M, \partial M ; n) \wedge Z^{\wedge n}\right) \\
& \simeq \Omega^{\infty}\left(\operatorname{Map}_{*}\left(M^{[n]}, \Sigma^{\infty}\left(\left(S^{m} Z\right)^{\wedge n}\right)\right)_{h \Sigma_{n}}\right) ;
\end{aligned}
$$

here $M^{[n]}=M^{\times n} / \Delta^{n} M$, where $\Delta^{n} M$ is the "fat diagonal", i.e. the space of $n$ tuples of points in $M$ such that at least two points coincide. The right hand side makes sense even if $M$ is not a manifold, and it suggests that (over a point)

$$
D_{n}\left(Q \operatorname{Map}_{*}(K, Y)\right) \simeq \Omega^{\infty}\left(\operatorname{Map}_{*}\left(K^{(n)}, \Sigma^{\infty} Y^{\wedge n}\right)_{h \Sigma_{n}}\right)
$$

(here $K^{(n)}=K^{\wedge n} / \Delta^{n} K$ ), which is, indeed, the case. Furthermore, since the action of $\Sigma_{n}$ on $K^{(n)}$ is free off the basepoint, and $K^{(n)}$ is a finite CW-complex, it follows, by a suitable version of the Adams isomorphism, that

$$
D_{n}\left(Q \operatorname{Map}_{*}(K, Y)\right) \simeq \Omega^{\infty}\left(\operatorname{Map}_{*}\left(K^{(n)}, \Sigma^{\infty} Y^{\wedge n}\right)^{\Sigma_{n}}\right) .
$$

The Taylor polynomials of a functor (as opposed to its homogeneous layers) are not easily retrievable from the Taylor polynomial of its composition with suspension. In particular, the fact that the Taylor tower of the functor $Y \mapsto Q \operatorname{Map}\left(M, S^{m} Y\right)$ splits does not imply that the Taylor tower of $Y \mapsto Q \operatorname{Map}(M, Y)$ splits (it doesn't). We write explicit formulas for the polynomial approximations of our functor in our main theorem (theorem 2) and prove that they are correct by establishing that they have the necessary universal properties. For example the quadratic approximation (still only over a point) is given by the following formula:

$$
P_{2} Q\left(\operatorname{Map}_{*}(K, Y)\right) \simeq \operatorname{Map}_{*}\left(K^{\wedge 2}, Q\left(Y^{\wedge 2}\right)\right)^{\Sigma_{2}}
$$

with the approximation map being the composition of the obvious maps

$$
Q \operatorname{Map}_{*}(K, Y) \rightarrow Q \operatorname{Map}_{*}\left(K^{\wedge 2}, Y^{\wedge 2}\right)^{\Sigma_{2}} \rightarrow \operatorname{Map}_{*}\left(K^{\wedge 2}, Q\left(Y^{\wedge 2}\right)\right)^{\Sigma_{2}}
$$

However, $P_{3} Q\left(\operatorname{Map}_{*}(K, Y)\right) \neq \operatorname{Map}_{*}\left(K^{\wedge 3}, Q\left(Y^{\wedge 3}\right)\right)^{\Sigma_{3}}$.

To pass from the Maclaurin tower to the general Taylor tower we observe that, given $M$ as in (0.1) and given two topological spaces $X$ and $Z$, the space of maps 
$\operatorname{Map}\left(M, X \vee_{x} S^{m} Z\right)$ can be approximated by means of certain "fiberwise configuration spaces" models, which stably splits. Thus the space $Q \operatorname{Map}\left(M, X \vee_{x} S^{m} Z\right)$ splits as follows:

$$
Q \operatorname{Map}\left(M, X \vee_{x} S^{m} Z\right) \simeq \prod_{n \geq 0} Q\left(\overline{C(M, \partial M ; n) \wedge_{x} \operatorname{Map}(M, X)_{+}} \wedge_{\Sigma_{n}} Z^{\wedge n}\right),
$$

where

$$
\begin{gathered}
\overline{C(M, \partial M ; n) \wedge_{x} \operatorname{Map}(M, X)_{+}} \\
=\left\{\left(m_{1} \ldots m_{n}\right) \wedge f \in C(M, \partial M ; n) \wedge \operatorname{Map}(M, X)_{+} \mid \forall 1 \leq i \leq n \quad f\left(m_{i}\right)=x\right\} .
\end{gathered}
$$

To "dualize" this formula we define certain functors, which we call fiberwise mapping spaces, and we show how to write the general Taylor tower in terms of these functors. Thus, for example,

$$
D_{n}\left(Q \operatorname{Map}_{*}(K, Y)\right) \simeq \overline{\operatorname{Map}_{*}\left(K^{(n)}, Q\left((Y / X)^{\wedge n} \wedge \operatorname{Map}_{*}(K, X)_{+}\right)\right)^{\Sigma_{n}}},
$$

where the right hand side denotes the space of maps satisfying a certain compatibility condition.

The paper is organized somewhat differently from the outline above: in section 1 , we define fiberwise mapping spaces. These spaces are defined as spaces which depend functorially on $K, X$ and $Y$. However, it is not clear from the definitions that these spaces are homotopy functors (of $Y$ or $K$ ), let alone excisive functors (of $Y)$.

In section 2, we prove a basic lemma about fiberwise mapping spaces, which in subsequent sections enables us to prove that fiberwise mapping spaces possess certain good properties.

In section 3, we use the lemma of section 2 to prove that certain fiberwise mapping spaces are, indeed, excisive homotopy functors of $Y$.

In section 4 , we use fiberwise mapping spaces to describe the Taylor tower of the functor $Q \operatorname{Map}_{*}(K, Y)$. In subsection 4.1 we state the main theorem. In particular, we describe the Taylor polynomials of $Q \operatorname{Map}_{*}(K, Y)$. This result appears to be new even in the case $X=*$, when fiberwise mapping spaces become ordinary mapping spaces. In subsection 4.2 we prove the main theorem.

The reader is strongly encouraged to proceed from the introduction directly to section 4.1, and refer to the earlier sections as need arises.

Remark 1. It is easy to see that, given any $(-1)$-connected spectrum $E$, our formulas can be generalized to describe the Taylor tower of the funcor

$$
Y \mapsto \Omega^{\infty}\left(E \wedge \operatorname{Map}_{*}(K, Y)\right) .
$$

Basically, one just replaces $Q$ with $\Omega^{\infty} E$ everywhere. Some care might be needed with the fiberwise case, but presumably it is not too hard to figure out. In particular, one gets a spectral sequence for the homology of the space of maps from a finite complex to a highly enough connected space. There is no doubt that many special cases of this spectral sequence are known. For instance, it was known to Goodwillie a long time ago that in the case $K=S^{1}$, one gets the usual EilenbergMoore spectral sequence for the homology of $\Omega X$. The spectral sequence probably has not been written in this generality before. However, the really new result of this paper is the functorial, explicit description of the extentions on the space level.

Acknowledgement: This is a much revised version of my Ph.D. thesis (Brown, 1993) written under the supervision of T. Goodwillie. 


\section{Fiberwise MAPPING SPACES}

Let $X$ be a fixed space with a basepoint. Throughout this paper, $T_{X}$ will denote the category of all spaces containing $\mathrm{X}$ as a retract. Thus an object of $T_{X}$ is a triple $(Y, \alpha, \rho)$ where $Y$ and $X$ are based spaces, $\alpha: Y \rightarrow X$ is a map, $\rho: X \rightarrow Y$ is a cofibration and the composite $X \stackrel{\rho}{\rightarrow} Y \stackrel{\alpha}{\rightarrow} X$ is the identity. A morphism $(Y, \alpha, \rho) \rightarrow\left(Y^{\prime}, \alpha^{\prime}, \rho^{\prime}\right)$ is a map $Y \rightarrow Y^{\prime}$ which commutes with $\alpha, \alpha^{\prime}, \rho$ and $\rho^{\prime}$ in the obvious sense. We consider the functor $Q \operatorname{Map}_{*}(K, Y)$, where $K$ is a finite CW complex with a basepoint, $Y \in T_{X}$ and $\operatorname{Map}_{*}(-,-)$ stands for the space of based maps. $Q(-)$ stands for $\Omega^{\infty} \Sigma^{\infty}(-)$.

For an integer $n \geq 1$, let $K_{1}^{n}$ be a $\Sigma_{n}$ equivariant subquotient of the space $K^{\times n}$. This means that there exist $\Sigma_{n}$ equivariant subcomplexes $K_{a} \subseteq K_{b}$ of $K^{\times n}$ such that $K_{1}^{n} \cong K_{b} / K_{a}$. We will denote by $P_{i}$ the projection of $K^{\times n}$ on its $i$-th coordinate. Obviously, if $K_{1}^{n}$ is not a subcomplex of $K^{\times n}$ then $P_{i}$ does not induce a map on $K_{1}^{n}$; however, it does induce a map on $K_{1}^{n} \backslash\{*\}$. We will denote this restricted map by the same symbol.

Let $W$ be a based space endowed with a map

$$
\alpha: W \backslash\{*\} \rightarrow X .
$$

In keeping with our conventions for maps induced by projections of cartesian products on summands, we define the maps

$$
P_{W i}: W^{\wedge n} \wedge \operatorname{Map}_{*}(K, X)_{+} \backslash\{*\} \rightarrow W
$$

(projection on $i^{t h}$ coordinate in $W^{n}$ ) and

$$
P_{F}: W^{\wedge n} \wedge \operatorname{Map}_{*}(K, X)_{+} \backslash\{*\} \rightarrow \operatorname{Map}_{*}(K, X)
$$

(projection on $\operatorname{Map}_{*}(K, X)$ ).

We are now ready to define fiberwise mapping spaces.

\section{Definition 1.}

$$
\begin{aligned}
& \overline{\operatorname{Map}_{*}\left(K_{1}^{n}, W^{\wedge n} \wedge \operatorname{Map}_{*}(K, X)_{+}\right)} \\
& =\left\{f \in \operatorname{Map}_{*}\left(K_{1}^{n}, W^{\wedge n} \wedge \operatorname{Map}_{*}(K, X)_{+}\right) \mid \forall k_{1}^{n} \in K_{1}^{n} \backslash f^{-1}(*)\right. \\
& \left.P_{F}\left(f\left(k_{1}^{n}\right)\right)\left(P_{i}\left(k_{1}^{n}\right)\right)=\alpha\left(P_{W i}\left(f\left(k_{1}^{n}\right)\right)\right) \forall 1 \leq i \leq n\right\} .
\end{aligned}
$$

We say that the space $\overline{\operatorname{Map}_{*}\left(K_{1}^{n}, W^{\wedge n} \wedge \operatorname{Map}_{*}(K, X)_{+}\right)}$is a fiberwise mapping space.

Likewise, we define the stable fiberwise mapping space

$$
\overline{\operatorname{Map}_{*}\left(K_{1}^{n}, Q\left(W^{\wedge n} \wedge \operatorname{Map}_{*}(K, X)_{+}\right)\right)}
$$

as follows:

\section{Definition 2.}

$$
\begin{aligned}
& \overline{\operatorname{Map}_{*}\left(K_{1}^{n}, Q\left(W^{\wedge n} \wedge \operatorname{Map}_{*}(K, X)_{+}\right)\right)} \\
& =\lim _{N}\left\{f \in \operatorname{Map}_{*}\left(S^{N} \wedge K_{1}^{n}, S^{N} \wedge\left(W^{\wedge n} \wedge \operatorname{Map}_{*}(K, X)_{+}\right)\right) \mid\right. \\
& \forall s^{N} \wedge k_{1}^{n} \in S^{N} \wedge K_{1}^{n} \backslash f^{-1}(*) \\
& \left.\quad P_{F}\left(f\left(s^{n} \wedge k_{1}^{n}\right)\right)\left(P_{i}\left(k_{1}^{n}\right)\right)=\alpha\left(P_{W i}\left(f\left(s^{n} \wedge k_{1}^{n}\right)\right)\right) \forall 1 \leq i \leq n\right\} .
\end{aligned}
$$

We will say that a function $f$ satisfies the bar condition if it is an element of some appropriate fiberwise mapping space.

Clearly, fiberwise mapping spaces are $\Sigma_{n}$ equivariant subspaces of the corresponding mapping spaces, where the action of $\Sigma_{n}$ is induced by the obvious action 
on $K_{1}^{n}$ and $W^{\wedge n}$ and the trivial action on $\operatorname{Map}_{*}(K, X)$. In particular, it makes sense to talk about maps which are equivariant and satisfy the bar condition. We will denote spaces of such maps by $\overline{\operatorname{Map}_{*}\left(K_{1}^{n}, W^{\wedge n} \wedge \operatorname{Map}_{*}(K, X)_{+}\right)^{\Sigma_{n}}}$, etc.

Notice that if $X \cong *$ then fiberwise mapping spaces are homeomorphic to ordinary mapping spaces. For instance, if $X \cong *$ then

$$
\overline{\operatorname{Map}_{*}\left(K_{1}^{n}, Q\left(W^{\wedge n} \wedge \operatorname{Map}_{*}(K, X)_{+}\right)\right)^{\Sigma_{n}}} \cong \operatorname{Map}_{*}\left(K_{1}^{n}, Q\left(W^{\wedge n}\right)\right)^{\Sigma_{n}}
$$

It is also clearly possible to define fiberwise mapping spaces such as

$$
\overline{\operatorname{Map}_{*}\left(K_{1}^{n}, V_{n} \wedge \operatorname{Map}_{*}(K, X)_{+}\right)^{\Sigma_{n}}}
$$

for any based space $V_{n}$ endowed with an action of $\Sigma_{n}$ and a $\Sigma_{n}$ equivariant map

$$
\tilde{\alpha}: V_{n} \backslash * \rightarrow X^{n} \text {. }
$$

\section{A FibRATION LEMMA}

At this point, it is not clear that fiberwise mapping spaces have good homotopy properties. We want to prove that certain fiberwise mapping spaces make excisive functors of $Y$. However it is not even obvious from the definition that they are homotopy functors of $Y, K$ and $K_{1}^{n}$. The following lemma will be the key tool for proving such statements, as it will enable us to make inductive proofs (on the dimension of $K_{1}^{n}$, on the number of cells in $K_{1}^{n}$, etc.). It will be referred to as the fibration lemma.

Let $Y \in T_{X}$. By definition, there is a cofibration $X \rightarrow Y$. Let $Y / X$ denote the quotient. Let $n \geq 1$. Let $K$ be a finite CW-complex. Let $K_{1}^{n}$ be a $\Sigma_{n}$ equivariant subquotient complex of $K^{\times n}$. Let $K_{2}^{n} \subseteq K_{1}^{n}$ be a $\Sigma_{n}$ equivariant subcomplex of $K_{1}^{n}$. Note that the map $\alpha: Y \rightarrow X$ induces a map $Y / X \backslash\{*\} \rightarrow X$.

The fibration lemma. Restriction maps such as

$$
\begin{gathered}
\overline{\operatorname{Map}_{*}\left(K_{1}^{n},(Y / X)^{\wedge n} \wedge \operatorname{Map}_{*}(K, X)_{+}\right)^{\Sigma_{n}}} \\
\frac{\downarrow}{\operatorname{Map}_{*}\left(K_{2}^{n},(Y / X)^{\wedge n} \wedge \operatorname{Map}_{*}(K, X)_{+}\right)^{\Sigma_{n}}}
\end{gathered}
$$

and

$$
\begin{aligned}
& \overline{\operatorname{Map}_{*}\left(K_{1}^{n}, Q\left((Y / X)^{\wedge n} \wedge \operatorname{Map}_{*}(K, X)_{+}\right)\right)^{\Sigma_{n}}} \\
& \frac{\downarrow}{\operatorname{Map}_{*}\left(K_{2}^{n}, Q\left((Y / X)^{\wedge n} \wedge \operatorname{Map}_{*}(K, X)_{+}\right)\right)^{\Sigma_{n}}}
\end{aligned}
$$

are Serre fibrations.

Proof. We will prove that the second map is a fibration, the proof of all other cases being identical. First, we prove the statement for the case when $K_{1}^{n}$ is a subcomplex of $K^{\times n}$. Following our conventions, we will denote by $P_{i}$ the projection of $K^{\times n}$ on its $i$-th coordinate. Again, by a slight abuse of notation, we also denote by $P_{i}$ its restrictions to $K_{1}^{n}$ and $K_{2}^{n}$. Our goal is to show that for every commutative square diagram of the form

$$
\begin{aligned}
& I^{m} \rightarrow \overline{\operatorname{Map}_{*}\left(K_{1}^{n}, Q\left((Y / X)^{\wedge n} \wedge \operatorname{Map}_{*}(K, X)_{+}\right)\right)^{\Sigma_{n}}} \\
& { }^{\downarrow} \times I \rightarrow \frac{\downarrow}{\operatorname{Map}_{*}\left(K_{2}^{n}, Q\left((Y / X)^{\wedge n} \wedge \operatorname{Map}_{*}(K, X)_{+}\right)\right)^{\Sigma_{n}}}
\end{aligned}
$$


there exists a map $I^{m} \times I \rightarrow \overline{\operatorname{Map}_{*}\left(K_{1}^{n}, Q\left((Y / X)^{\wedge n} \wedge \operatorname{Map}_{*}(K, X)_{+}\right)\right)^{\Sigma_{n}}}$, which commutes with all the maps in the square. This is equivalent to showing that for every diagram of based map of the form

$$
\begin{aligned}
& \left(I^{m} \times I\right)_{+} \wedge K_{1}^{n} \quad Q\left((Y / X)^{\wedge n} \wedge \operatorname{Map}_{*}(K, X)_{+}\right) \\
& I_{+}^{m} \wedge\left(I_{+} \wedge K_{2}^{n} \cup\{0\}_{+} \wedge K_{1}^{n}\right)
\end{aligned}
$$

where the map $T$ is equivariant and satisfies the bar condition, there exists a based map $U:\left(I^{m} \times I\right)_{+} \wedge K_{1}^{n} \rightarrow Q\left((Y / X)^{\wedge n} \wedge \operatorname{Map}_{*}(K, X)_{+}\right)$which is equivariant, satisfies the bar condition, and completes the diagram to a commutative triangle. Since $\left(K_{1}^{n}, K_{2}^{n}\right)$ is a $\Sigma_{n}$-NDR pair, it follows that $\left(I_{+} \wedge K_{1}^{n}, I_{+} \wedge K_{2}^{n} \cup\{0\}_{+} \wedge K_{1}^{n}\right)$ is a $\Sigma_{n}$-DR pair. Hence there exists a $\Sigma_{n}$ equivariant retraction

$$
G: I_{+} \wedge K_{1}^{n} \rightarrow I_{+} \wedge K_{2}^{n} \cup\{0\}_{+} \wedge K_{1}^{n}
$$

Therefore, we have the following diagram:

$$
\begin{aligned}
& I_{+}^{m} \wedge I_{+} \wedge K_{1}^{n} \quad Q\left((Y / X)^{\wedge n} \wedge \operatorname{Map}_{*}(K, X)_{+}\right) \\
& I_{+}^{m} \wedge\left(I_{+} \wedge K_{2}^{n} \cup\{0\}_{+} \wedge K_{1}^{n}\right)
\end{aligned}
$$

One could consider the map $T \circ\left(I d_{I^{m}} \times G\right)$ as the first candidate for being the map $U$ above. It is, indeed, $\Sigma_{n}$ equivariant, but it does not satisfy the bar condition. To rectify that, we need the following proposition.

Proposition 1. There exists a based map $H: I_{+} \wedge K_{1}^{n} \rightarrow \operatorname{Map}_{*}(K, K)$ with the following properties:

(1) $\left.H\right|_{I_{+} \wedge K_{2}^{n} \cup\{0\}_{+} \wedge K_{1}^{n}} \equiv I d \in \operatorname{Map}_{*}(K, K)$.

(2) $H\left(k_{1}^{n}, t\right)\left(P_{i}\left(k_{1}^{n}\right)\right)=P_{i}\left(P_{K_{1}^{n}}\left(G\left(k_{1}^{n}, t\right)\right)\right) \quad \forall 1 \leq i \leq n, \forall k_{1}^{n} \in K_{1}^{n}, \forall t \in I$

$\left(P_{K_{1}^{n}}\right.$ stands for the obvious projection of $I_{+} \wedge K_{1}^{n}$ on $\left.K_{1}^{n}\right)$.

(3) $H$ is $\Sigma_{n}$ equivariant.

Note that we take the basepoint of $\operatorname{Map}_{*}(K, K)$ to be the identity map.

Proof. Our assertion is equivalent to the one stating that there exists a based map

$$
H^{*}: I_{+} \wedge K \wedge K_{1}^{n} \rightarrow K
$$

which satisfies the following conditions:

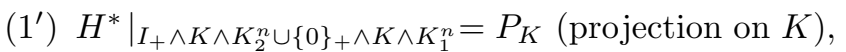

$\left.\left(2^{\prime}\right) H^{*}\right|_{I_{+} \wedge \Delta^{i} K}=P_{i}\left(P_{K_{1}^{n}}\left(G\left(P_{I_{+} \wedge K_{1}^{n}}\right)\right)\right) \quad \forall 1 \leq i \leq n, \quad \forall k_{1}^{n} \in K_{1}^{n}, \quad \forall t \in I$ $\left(\Delta^{i} K=\left\{\left(k \wedge k_{1}^{n}\right) \in K \wedge K_{1}^{n} \mid\right.\right.$ if $k \wedge k_{1}^{n} \neq\{$ basepoint $\}$ then $\left.\left.P_{i}\left(k_{1}^{n}\right)=k\right\}\right)$,

$\left(3^{\prime}\right) H^{*}$ is $\Sigma_{n}$ equivariant.

The first thing we need to check is that these conditions are compatible. Condition $\left(1^{\prime}\right)$ is compatible with any of the conditions in $\left(2^{\prime}\right)$ because $G$ is a retraction. Condition $\left(1^{\prime}\right)$ is compatible with $\left(3^{\prime}\right)$ because projection on $K$ is $\Sigma_{n}$ equivariant. Any of the conditions in $\left(2^{\prime}\right)$ is compatible with $\left(3^{\prime}\right)$ because $G$ is $\Sigma_{n}$ equivariant. Finally, it is obvious that all the conditions in $\left(2^{\prime}\right)$ are compatible with each other.

Thus, $H^{*}$ is well defined on the subspace

$$
I_{+} \wedge\left(K \wedge K_{2}^{n} \cup\left(\bigcup_{i=1}^{i=n} \Delta^{i} K\right)\right) \cup\{0\}_{+} \wedge K \wedge K_{1}^{n}
$$


of $I_{+} \wedge K \wedge K_{1}^{n}$. The pair

$$
\left(K \wedge K_{1}^{n},\left(K \wedge K_{2}^{n} \cup\left(\bigcup_{i=1}^{i=n} \Delta^{i} K\right)\right)\right)
$$

is a $\Sigma_{n}$ NDR pair, and so the pair

$$
\left(I_{+} \wedge K \times K_{1}^{n}, I_{+} \wedge\left(K \wedge K_{2}^{n} \cup\left(\bigcup_{i=1}^{i=n} \Delta^{i} K\right)\right) \cup\{0\}_{+} \wedge K \wedge K_{1}^{n}\right)
$$

is a $\Sigma_{n}$ DR pair. Therefore $H^{*}$ can be extended to $I_{+} \wedge K \wedge K_{1}^{n}$.

Now it is easy to see that the map $H$ induces a ( $\Sigma_{n}$ equivariant) map

$$
\tilde{H}:\left(I^{m} \times I\right)_{+} \wedge K_{1}^{n} \rightarrow \operatorname{Map}_{*}\left(\operatorname{Map}_{*}(K, X), \operatorname{Map}_{*}(K, X)\right)
$$

defined as the composition

$$
\left(I^{m} \times I\right)_{+} \wedge K_{1}^{n} \rightarrow I_{+} \wedge K_{1}^{n} \stackrel{H}{\rightarrow} \operatorname{Map}_{*}(K, K) \rightarrow \operatorname{Map}_{*}\left(\operatorname{Map}_{*}(K, X), \operatorname{Map}_{*}(K, X)\right) .
$$

The map $\tilde{H}$ in turn induces a ( $\Sigma_{n}$ equivariant) map

$$
\begin{aligned}
Q \hat{H}: & \left(I^{m} \times I\right)_{+} \wedge K_{1}^{n} \\
& \rightarrow \operatorname{Map}_{*}\left(Q\left((Y / X)^{\wedge n} \wedge \operatorname{Map}_{*}(K, X)_{+}\right), Q\left((Y / X)^{\wedge n} \wedge \operatorname{Map}_{*}(K, X)_{+}\right)\right),
\end{aligned}
$$

which in turn induces a map $U$ as follows:

$$
\begin{aligned}
U:\left(I^{m} \times I\right)_{+} \wedge K_{1}^{n} & \rightarrow Q\left((Y / X)^{\wedge n} \wedge \operatorname{Map}_{*}(K, X)_{+}\right), \\
\left(v, t, k_{1}^{n}\right) & \mapsto Q \hat{H}\left(v, t, k_{1}^{n}\right)\left(T \circ\left(I d_{I_{+}^{m}} \wedge G\right)\left(v, t, k_{1}^{n}\right)\right) .
\end{aligned}
$$

In order to show that the map $U$ has all the desired properties we need to check that:

$\left.1^{\prime \prime}\right)\left.U\right|_{\left(I^{m} \times I\right)_{+} \wedge K_{2}^{n} \cup\left(I^{m} \times\{0\}\right)_{+} \wedge K_{1}^{n}=T .}$.

$\left.2^{\prime \prime}\right) U$ satisfies the bar condition.

$\left.3^{\prime \prime}\right) U$ is $\Sigma_{n}$ equivariant.

$\left(1^{\prime \prime}\right)$ follows from condition (1) in Proposition 1 and from the fact that $G$ is a retraction. ( $\left.2^{\prime \prime}\right)$ follows from condition (2) in Proposition 1 and $\left(3^{\prime \prime}\right)$ follows from the fact that $Q \hat{H}, T$ and $G$ are equivariant maps.

We have proved the lemma for the case when $K_{1}^{n}$ is a subcomplex of $K^{\times n}$. Now assume that $K_{1}^{n} \cong \widetilde{K_{1}^{n}} / K_{0}$ and $K_{2}^{n} \cong \widetilde{K_{2}^{n}} / K_{0}$, where $K_{0} \subseteq \widetilde{K_{2}^{n}} \subseteq \widetilde{K_{1}^{n}}$ are $\Sigma_{n}$ equivariant subcomplexes of $K^{\times n}$, and consider the diagram

$\overline{\operatorname{Map}_{*}\left(K_{1}^{n}, Q\left((Y / X)^{\wedge n} \wedge \operatorname{Map}_{*}(K, X)_{+}\right)\right)^{\Sigma_{n}}} \rightarrow \overline{\operatorname{Map}_{*}\left(\widetilde{K_{1}^{n}}, Q\left((Y / X)^{\wedge n} \wedge \operatorname{Map}_{*}(K, X)_{+}\right)\right)^{\Sigma_{n}}}$
$\frac{\downarrow}{\operatorname{Map}_{*}\left(K_{2}^{n}, Q\left((Y / X)^{\wedge n} \wedge \operatorname{Map}_{*}(K, X)_{+}\right)\right)^{\Sigma_{n}}} \rightarrow \overline{\operatorname{Map}_{*}\left(\widetilde{K_{2}^{n}}, Q\left((Y / X)^{\wedge n} \wedge \operatorname{Map}_{*}(K, X)_{+}\right)\right)^{\Sigma_{n}}}$

It is very easy to check that this diagram is a strict pullback, and we have proved that the right map is a fibration. Therefore, the left map is a fibration.

Corollary 1. The homotopy fiber of the restriction map

$$
\begin{aligned}
& \overline{\operatorname{Map}_{*}\left(K_{1}^{n}, Q\left((Y / X)^{\wedge n} \wedge \operatorname{Map}_{*}(K, X)_{+}\right)\right)^{\Sigma_{n}}} \\
& \frac{\downarrow}{\operatorname{Map}_{*}\left(K_{2}^{n}, Q\left((Y / X)^{\wedge n} \wedge \operatorname{Map}_{*}(K, X)_{+}\right)\right)^{\Sigma_{n}}}
\end{aligned}
$$


is homotopy equivalent to

$$
\overline{\operatorname{Map}_{*}\left(K_{1}^{n} / K_{2}^{n}, Q\left((Y / X)^{\wedge n} \wedge \operatorname{Map}_{*}(K, X)_{+}\right)\right)^{\Sigma_{n}}} .
$$

\section{Properties of FiberWise mapping SPACES}

3.1. Fiberwise mapping spaces are homotopy functors of $K_{1}^{n}$. As a first step towards analyzing fiberwise mapping spaces, we have to prove that they are homotopy functors of $K_{1}^{n}$ in some suitable sense. The following lemma will suffice for our needs.

Lemma 1. Let $K_{1}^{n}$ be a $\Sigma_{n}$ equivariant subquotient of $K^{\times n}$. Let $K_{2}^{n}$ be a $\Sigma_{n}$ equivariant deformation retract of $K_{1}^{n}$. Then the restriction map

$$
\begin{gathered}
\overline{\operatorname{Map}_{*}\left(K_{1}^{n}, Q\left((Y / X)^{\wedge n} \wedge \operatorname{Map}_{*}(K, X)_{+}\right)\right)^{\Sigma_{n}}} \\
\frac{\downarrow r}{\operatorname{Map}_{*}\left(K_{2}^{n}, Q\left((Y / X)^{\wedge n} \wedge \operatorname{Map}_{*}(K, X)_{+}\right)\right)^{\Sigma_{n}}}
\end{gathered}
$$

is a homotopy equivalence.

Proof. The proof is very similar to the proof of the fibration lemma. First we assume that $K_{1}^{n}$ is a subcomplex of $K^{\times n}$. We will construct a map

$$
\begin{aligned}
s: & \overline{\operatorname{Map}_{*}\left(K_{2}^{n}, Q\left((Y / X)^{\wedge n} \wedge \operatorname{Map}_{*}(K, X)_{+}\right)\right)^{\Sigma_{n}}} \rightarrow \\
& \rightarrow \overline{\operatorname{Map}_{*}\left(K_{1}^{n}, Q\left((Y / X)^{\wedge n} \wedge \operatorname{Map}_{*}(K, X)_{+}\right)\right)^{\Sigma_{n}}}
\end{aligned}
$$

and prove that it is a two-sided homotopy inverse of $r$.

By our assumption on $K_{2}^{n}$, there exists a homotopy

$$
G: I_{+} \wedge K_{1}^{n} \rightarrow K_{1}^{n}
$$

such that

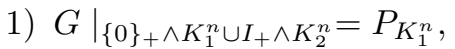

2) $\left.G\right|_{\{1\}_{+} \wedge K_{2}^{n}}\left(K_{1}^{n}\right) \subseteq K_{2}^{n}$,

3) $G$ is $\Sigma_{n}$-equivariant.

Proposition 2. There exists a homotopy

$$
H: I_{+} \wedge K_{1}^{n} \rightarrow \operatorname{Map}_{*}(K, K)
$$

such that

1') $\left.H\right|_{\{0\}_{+} \wedge K_{1}^{n} \cup I_{+} \wedge K_{2}^{n}} \equiv I d_{K}$,

2') $H\left(t, k_{1}^{n}\right)\left(P_{i}\left(k_{1}^{n}\right)\right)=P_{i}\left(G\left(t, k_{1}^{n}\right)\right), i=1, \ldots, n$,

$\left.3^{\prime}\right) H$ is $\Sigma_{n}$-equivariant.

Proof. The proof is virtually identical to the proof of proposition 1.

Now define a homotopy

$$
\begin{aligned}
\tilde{s} & : I_{+} \wedge \operatorname{Map}_{*}\left(K_{1}^{n}, Q\left((Y / X)^{\wedge n} \wedge \operatorname{Map}_{*}(K, X)_{+}\right)\right)^{\Sigma_{n}} \\
& \rightarrow \operatorname{Map}_{*}\left(K_{1}^{n}, Q\left((Y / X)^{\wedge n} \wedge \operatorname{Map}_{*}(K, X)_{+}\right)\right)^{\Sigma_{n}}
\end{aligned}
$$

as follows: first, we observe that a map as above is exactly the same as a map

$$
\begin{aligned}
\operatorname{Map}_{*} & \left(K_{1}^{n}, Q\left((Y / X)^{\wedge n} \wedge \operatorname{Map}_{*}(K, X)_{+}\right)\right)^{\Sigma_{n}} \\
& \rightarrow \operatorname{Map}_{*}\left(I_{+} \wedge K_{1}^{n}, Q\left((Y / X)^{\wedge n} \wedge \operatorname{Map}_{*}(K, X)_{+}\right)\right)^{\Sigma_{n}} .
\end{aligned}
$$


Such a map is induced by $G$. We compose this map with a map

$$
\begin{aligned}
\operatorname{Map}_{*} & \left(I_{+} \wedge K_{1}^{n}, Q\left((Y / X)^{\wedge n} \wedge \operatorname{Map}_{*}(K, X)_{+}\right)\right)^{\Sigma_{n}} \\
& \rightarrow \operatorname{Map}_{*}\left(I_{+} \wedge K_{1}^{n}, Q\left((Y / X)^{\wedge n} \wedge \operatorname{Map}_{*}(K, X)_{+}\right)\right)^{\Sigma_{n}}
\end{aligned}
$$

induced by $H$ in the obvious way. Thus $\tilde{s}$ is induced by the composition of these two maps. Let $s$ be the map obtained by restricting $\tilde{s}$ to

$$
\{1\}_{+} \wedge \operatorname{Map}_{*}\left(K_{1}^{n}, Q\left((Y / X)^{\wedge n} \wedge \operatorname{Map}_{*}(K, X)_{+}\right)\right)^{\Sigma_{n}} .
$$

Thus $s$ is a map

$$
\begin{aligned}
\operatorname{Map}_{*} & \left(K_{1}^{n}, Q\left((Y / X)^{\wedge n} \wedge \operatorname{Map}_{*}(K, X)_{+}\right)\right)^{\Sigma_{n}} \\
& \rightarrow \operatorname{Map}_{*}\left(K_{1}^{n}, Q\left((Y / X)^{\wedge n} \wedge \operatorname{Map}_{*}(K, X)_{+}\right)\right)^{\Sigma_{n}} .
\end{aligned}
$$

Because of condition (2) in the definition of $G, s$ may in fact be considered as a map

$$
\begin{aligned}
\operatorname{Map}_{*} & \left(K_{2}^{n}, Q\left((Y / X)^{\wedge n} \wedge \operatorname{Map}_{*}(K, X)_{+}\right)\right)^{\Sigma_{n}} \\
& \rightarrow \operatorname{Map}_{*}\left(K_{1}^{n}, Q\left((Y / X)^{\wedge n} \wedge \operatorname{Map}_{*}(K, X)_{+}\right)\right)^{\Sigma_{n}}
\end{aligned}
$$

Moreover, it follows from property (2) in the definition of $H$ that $s$ preserves the bar condition, i.e

$$
\begin{aligned}
s: & \left(\overline{\operatorname{Map}_{*}\left(K_{2}^{n}, Q\left((Y / X)^{\wedge n} \wedge \operatorname{Map}_{*}(K, X)_{+}\right)\right)^{\Sigma_{n}}}\right) \\
& \subseteq\left(\overline{\operatorname{Map}_{*}\left(K_{1}^{n}, Q\left((Y / X)^{\wedge n} \wedge \operatorname{Map}_{*}(K, X)_{+}\right)\right)^{\Sigma_{n}}}\right) .
\end{aligned}
$$

Therefore we may think of $s$ as a map

$$
\begin{aligned}
s: & \overline{\operatorname{Map}_{*}\left(K_{2}^{n}, Q\left((Y / X)^{\wedge n} \wedge \operatorname{Map}_{*}(K, X)_{+}\right)\right)^{\Sigma_{n}}} \\
& \rightarrow \overline{\operatorname{Map}_{*}\left(K_{1}^{n}, Q\left((Y / X)^{\wedge n} \wedge \operatorname{Map}_{*}(K, X)_{+}\right)\right)^{\Sigma_{n}}}
\end{aligned}
$$

It remains to check that $s$ is a two-sided homotopy inverse of $r$. Obviously, $r s=\mathrm{Id}$, since $\left.G\right|_{I_{+} \wedge K_{2}}=P_{K_{1}}$ and $\left.H\right|_{I_{+} \wedge K_{2}} \equiv \operatorname{Id}_{K}$. To see that $s r \simeq \mathrm{Id}$, observe that $\tilde{s}$ induces the required homotopy. We have proved the lemma in the case when $K_{1}$ is a subcomplex of $K^{\times n}$. The general case is concluded in the same way as in the last step of the proof of the fibration lemma.

3.2. Fiberwise mapping spaces make excisive homotopy functors of $Y$. Now we have set the ground to prove that fiberwise mapping spaces have some good properties.

Let $n \geq 1$. Let $Y \in T_{X}$. Let $K_{1}^{n}$ be as usual. Consider the functor

$$
\overline{\operatorname{Map}_{*}\left(K_{1}^{n}, Q\left((Y / X)^{\wedge n} \wedge \operatorname{Map}_{*}(K, X)_{+}\right)\right)^{\Sigma_{n}}}
$$

Theorem 1. This functor is

1) a homotopy functor of $Y \rightarrow X$. That is, if a map $Y \rightarrow Y^{\prime}$ is a weak equivalence in $T_{X}$, then the induced map

$$
\begin{aligned}
& \overline{\operatorname{Map}_{*}\left(K_{1}^{n}, Q\left((Y / X)^{\wedge n} \wedge \operatorname{Map}_{*}(K, X)_{+}\right)\right)^{\Sigma_{n}}} \\
& \rightarrow \overline{\operatorname{Map}_{*}\left(K_{1}^{n}, Q\left(\left(Y^{\prime} / X\right)^{\wedge n} \wedge \operatorname{Map}_{*}(K, X)_{+}\right)\right)^{\Sigma_{n}}}
\end{aligned}
$$

is a weak equivalence.

2) excisive of degree $n$. Moreover, if the action of $\Sigma_{n}$ on $K_{1}^{n}$ is free off the basepoint, then the functor is homogeneous of degree $n$. 
3) a reduced functor of $T_{X}$.

Proof of the theorem. First, let us discuss statement (3). It says that if $Y \simeq X$ then

$$
\overline{\operatorname{Map}_{*}\left(K_{1}^{n}, Q\left((Y / X)^{\wedge n} \wedge \operatorname{Map}_{*}(K, X)_{+}\right)\right)^{\Sigma_{n}}} \simeq * .
$$

Assuming that statement (1) is true, it is enough to prove (3) for the case $Y \cong X$. But in this case $Y / X \cong *$ and therefore the space

$$
\overline{\operatorname{Map}_{*}\left(K_{1}^{n}, Q\left((Y / X)^{\wedge n} \wedge \operatorname{Map}_{*}(K, X)_{+}\right)\right)^{\Sigma_{n}}}
$$

is a subspace of the space of maps of $K_{1}^{n}$ into a one point space, which is also a one

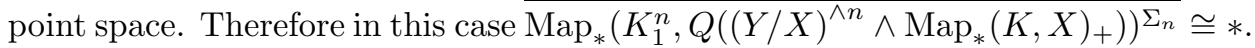

The proof of statements (1) and (2) is by induction on $d$, the dimension of $K_{1}^{n}$. We start with the case $d=0$, i.e. $K_{1}^{n}$ is a finite based set with an action of $\Sigma_{n}$. Let $e$ be the number of orbits under this action not counting the orbit consisting of the basepoint. We proceed with an induction on $e$. So, we first prove the case $e=1$. In this case $K_{1}^{n}$ is a $\Sigma_{n}$ orbit of one point in $K^{\times n}$ plus the basepoint. Choose a representative point $\left(k_{1}, \ldots, k_{n}\right)$ in $K_{1}^{n}$. Let $\widetilde{K_{1}^{n}}$ be the set consisting of this representative and the basepoint. Let $S$ be the set of distinct elements in $\left(k_{1}, \ldots, k_{n}\right)$. Let $s$ be the cardinality of $S$, let $\widetilde{K_{1}^{s}}$ be the the set consisting of the $s$-tuple $\left(k_{1}, \ldots, k_{s}\right)$ and the basepoint. Then, recalling the definitions, it is clear that

$$
\begin{aligned}
& \overline{\operatorname{Map}_{*}\left(K_{1}^{n}, Q\left((Y / X)^{\wedge n} \wedge \operatorname{Map}_{*}(K, X)_{+}\right)\right)^{\Sigma_{n}}} \\
& \cong \widetilde{\operatorname{Map}_{*}\left(\widetilde{K_{1}^{s}}, Q\left((Y / X)^{\wedge s} \wedge \operatorname{Map}_{*}(K, X)_{+}\right)\right)}
\end{aligned}
$$

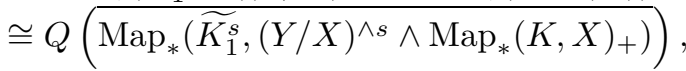

and we will denote the space

$$
\overline{\operatorname{Map}_{*}\left(\widetilde{K_{1}^{s}},(Y / X)^{\wedge s} \wedge \operatorname{Map}_{*}(K, X)_{+}\right)}
$$

simply by $\overline{(Y / X)^{\wedge s} \wedge \operatorname{Map}_{*}(K, X)_{+}}$. Explicitly, this is the following space:

$$
\begin{aligned}
& \overline{\left((Y / X)^{\wedge s} \wedge \operatorname{Map}_{*}(K, X)_{+}\right)} \\
& =\left\{\left(\overline{y_{1}} \wedge \ldots \wedge \overline{y_{s}}, f\right) \in(Y / X)^{\wedge s} \wedge \operatorname{Map}_{*}(K, X)_{+} \mid\right. \\
& \text {either }\left(\overline{y_{1}} \wedge \ldots \wedge \overline{y_{s}}\right) \text { is the basepoint or } \\
& \left.\quad \forall 1 \leq i \leq s f\left(k_{i}\right)=\alpha\left(y_{i}\right)\right\} .
\end{aligned}
$$

We define $\overline{(Y / X)^{\wedge s} \times \operatorname{Map}_{*}(K, X)}$ similarly to $\overline{(Y / X)^{\wedge s} \wedge \operatorname{Map}_{*}(K, X)_{+}}$, and it is straightforward to check that

$$
\overline{(Y / X)^{\wedge s} \wedge \operatorname{Map}_{*}(K, X)_{+}} \cong \frac{\overline{(Y / X)^{\wedge s} \times \operatorname{Map}_{*}(K, X)}}{* \times \operatorname{Map}_{*}(K, X)} .
$$

Likewise, it is possible to define the space

$$
\overline{(Y / X)^{\wedge s} \times \operatorname{Map}_{*}\left(K_{0}, X\right)}
$$

for any $K_{0}$ such that $S \subseteq K_{0} \subseteq K$. In particular, it is defined for $S=K_{0}$. Now consider the commutative square

$$
\begin{array}{ccc}
\overline{(Y / X)^{\wedge s} \times \operatorname{Map}_{*}(K, X)} & \rightarrow \operatorname{Map}_{*}(K, X) \\
\frac{\downarrow}{(Y / X)^{\wedge s} \times \operatorname{Map}_{*}(S, X)} & \rightarrow \operatorname{Map}_{*}(S, X)
\end{array}
$$


Proposition 3. This is a strict pullback square.

Proof. Easy.

We conclude that since the right vertical map map is a fibration, the left vertical map is a fibration too.

Recall that our goal was to prove that the functor $Q\left(\overline{(Y / X)^{\wedge s} \wedge \operatorname{Map}_{*}(K, X)_{+}}\right)$ is an excisive homotopy functor (and in case the action of $\Sigma_{n}$ on $K_{1}^{n}$ is free off the basepoint, is also homogeneous of degree $n$ ). Since $Q$ takes co-Cartesian cubes to Cartesian cubes, it is enough to prove that the functor $\left(\overline{(Y / X)^{\wedge s} \wedge \operatorname{Map}_{*}(K, X)_{+}}\right)$ is a homology functor, which satisfies the "homology limit axiom", which takes strongly co-Cartesian $n+1$-cubes to co-Cartesian $n+1$-cubes (and which, in case the action of $\Sigma_{n}$ on $K_{1}^{n}$ is free off the basepoint, also takes $l$-connected spaces over $X$ to $n l$-connected spaces $)$. We will show that a) the functor $\left(\overline{(Y / X)^{\wedge s} \times \operatorname{Map}_{*}(S, X)}\right)$ has all the required properties, and b) that this implies the same for

$$
\left(\overline{(Y / X)^{\wedge s} \times \operatorname{Map}_{*}(K, X)}\right) .
$$

Indeed, to see (a) notice that there is a cofibration sequence

$$
\operatorname{Map}_{*}(S, X) \rightarrow\left(\overline{(Y / X)^{\wedge s} \times \operatorname{Map}_{*}(S, X)}\right) \rightarrow(Y / X)^{\wedge s} .
$$

To see (b) recall that there is a fibration sequence

$$
\operatorname{Map}_{*}(K / S, X) \rightarrow\left(\overline{(Y / X)^{\wedge s} \times \operatorname{Map}_{*}(K, X)}\right) \rightarrow\left(\overline{(Y / X)^{\wedge s} \times \operatorname{Map}_{*}(S, X)}\right)
$$

in which the fiber is constant (as a functor of $Y$ ). The statement of (b) is easily implied by the following two well known propositions:

Proposition 4. Let

$$
\begin{array}{cccccc}
Y_{0} & \rightarrow & Y_{1} & \rightarrow & Y_{2} & \rightarrow \ldots \\
\downarrow & & \downarrow & & \downarrow & \\
X_{0} & \rightarrow & X_{1} & \rightarrow & X_{2} & \rightarrow \ldots
\end{array}
$$

be a commutative diagram such that each square is homotopy Cartesian. Then the homotopy fiber of the induced map on the mapping telescopes is the same as of the maps $Y_{i} \rightarrow X_{i}$.

Proof. [10, Lemma 1.8]

Proposition 5. Let $p: E \rightarrow B$ be a map of filtered spaces such that $F_{n} E=$ $p^{-1} F_{n} B$ for $n \geq 0$ and, for $n \geq 1, p: F_{n} E \rightarrow F_{n} B$ is obtained by passage to pushouts from a commutative diagram of the form

$$
\begin{array}{ccccc}
F_{n-1} E & \stackrel{g_{n}}{\leftarrow} & D_{n} & \stackrel{j_{n}}{\longrightarrow} & E_{n} \\
p \downarrow & & \downarrow_{n} & & \downarrow_{n} \\
F_{n-1} B & \stackrel{f_{n}}{\leftarrow} & A_{n} & \stackrel{i_{n}}{\longrightarrow} & B_{n}
\end{array}
$$

Suppose that the following conditions hold.

(i) $p \mid F_{0} E$ is a quasifibration.

(ii) Each map $p_{n}: E_{n} \rightarrow B_{n}$ is a fibration.

(iii) Each map $i_{n}: A_{n} \rightarrow B_{n}$ is a cofibration.

(iv) Each right square is a pullback. 
(v) $g_{n}$ induces weak equivalences on all fibers.

Then $p: e \rightarrow B$ is a quasifibration, and so are its restrictions to $F_{n} E$ for all $n \geq 0$.

Proof. [8, Theorem 2.7]

It remains to prove that if the action of $\Sigma_{n}$ on $K_{1}^{n}$ is free off the basepoint, then the functor

$$
Q\left(\overline{(Y / X)^{\wedge s} \wedge \operatorname{Map}_{*}(K, X)_{+}}\right)
$$

is homogeneous of degree $n$, for which, since we have shown that this functor is $n$ excisive, it is enough to show that it takes $l$-connected spaces over $X$ to $n l$-connected spaces. To prove this it is enough to prove that the map

$$
\left(\overline{(Y / X)^{\wedge s} \times \operatorname{Map}_{*}(K, X)}\right) \rightarrow \operatorname{Map}_{*}(K, X)
$$

is $n l$-connected if $(Y / X)$ is $l$-connected. If $\Sigma_{n}$ is acting freely (off the basepoint) on $K_{1}^{n}$ then $s=n$, and (again) there is a pullback square

$$
\begin{array}{ccc}
\overline{(Y / X)^{\wedge n} \times \operatorname{Map}_{*}(K, X)} & \rightarrow \operatorname{Map}_{*}(K, X) \\
\bar{\downarrow} & \downarrow \\
(Y / X)^{\wedge n} \times \operatorname{Map}_{*}(S, X) & \rightarrow \operatorname{Map}_{*}(S, X)
\end{array}
$$

We need to prove that a certain connectivity property holds for the top horizontal map. But the condition clearly holds for the bottom map, and therefore for the top map.

We have completed the proof of the case $e=1$. Now let $K_{1}^{n}$ have $e+1$ orbits (not counting the basepoint) and assume that in the case of $e$ orbits the theorem is true. Let $K_{2}^{n} \subseteq K_{1}^{n}$ be a subspace containing one orbit and the basepoint. Clearly, the quotient $K_{1}^{n} / K_{2}^{n}$ is the union of $e$ orbits and the basepoint. Consider the sequence

$$
\begin{gathered}
\overline{\operatorname{Map}_{*}\left(K_{1}^{n} / K_{2}^{n}, Q\left((Y / X)^{\wedge n} \wedge \operatorname{Map}_{*}(K, X)_{+}\right)\right)^{\Sigma_{n}}} \\
\frac{\downarrow}{\operatorname{Map}_{*}\left(K_{1}^{n}, Q\left((Y / X)^{\wedge n} \wedge \operatorname{Map}_{*}(K, X)_{+}\right)\right)^{\Sigma_{n}}} \\
\frac{\downarrow}{\operatorname{Map}_{*}\left(K_{2}^{n}, Q\left((Y / X)^{\wedge n} \wedge \operatorname{Map}_{*}(K, X)_{+}\right)\right)^{\Sigma_{n}}}
\end{gathered}
$$

It follows immediately from the fibration lemma and its corollary that this is a fibration sequence, and by the induction assumption, the theorem is true for the base and the fiber. It follows that it is true for the total space.

We have completed the case $d=0$. Now let $K_{1}^{n}$ be of dimension $d+1$, and assume that the theorem is true if $K_{1}^{n}$ is of dimension $d$. Let $\widetilde{X}_{d+1}$ be the disjoint union of the $d+1$-cells in $K_{1}^{n}$. So, $\widetilde{X}_{d+1}$ is a disjoint union of $d+1$-dimensional balls. For each of these balls take an open "subball" of some fixed slightly smaller radius. Let $X_{d+1}$ be the disjoint union of these subballs and let $\bar{X}_{d+1}$ be the union of the corresponding closed balls. Let ${ }_{d} K_{1}^{n}=K_{1}^{n} \backslash X_{d+1}$. Clearly, the $d$-skeleton of $K_{1}^{n}$ is a $\Sigma_{n}$-equivariant homotopy retract of ${ }_{d} K_{1}^{n}$. There is an equivariant homotopy pushout square

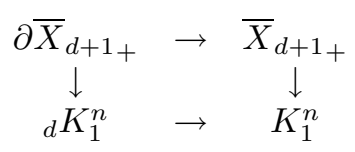


It follows immediately from the fibration lemma that it induces a homotopy pullback square

$$
\begin{aligned}
& \overline{\operatorname{Map}_{*}\left(K_{1}^{n}, Q\left((Y / X)^{\wedge n} \wedge \operatorname{Map}_{+}(K, X)_{+}\right)\right)^{\Sigma_{n}}} \\
& \frac{\downarrow}{\operatorname{Map}_{*}\left({ }_{d} K_{1}^{n}, Q\left((Y / X)^{\wedge n} \wedge \operatorname{Map}_{+}(K, X)_{+}\right)\right)^{\Sigma_{n}}} \\
& \rightarrow \overline{\operatorname{Map}_{*}\left(\bar{X}_{d+1_{+}}, Q\left((Y / X)^{\wedge n} \wedge \operatorname{Map}_{*}(K, X)_{+}\right)\right)^{\Sigma_{n}}} \\
& \rightarrow \frac{\downarrow}{\operatorname{Map}_{*}\left(\partial \bar{X}_{d+1_{+}}, Q\left((Y / X)^{\wedge n} \wedge \operatorname{Map}_{*}(K, X)_{+}\right)\right)^{\Sigma_{n}}}
\end{aligned}
$$

The theorem holds for the lower left and lower right corners by our induction assumption. The same is true for the upper right corner by lemma 1 since $\bar{X}_{d+1+}$ is equivariantly equivalent to a finite set. Therefore the theorem is true for the functor at the upper left corner. This completes the induction and the proof of the theorem.

\section{Description of the TAYlor tower}

4.1. The main theorem. In this section we will define a sequence of functors $P_{X}^{n} Q \operatorname{Map}_{*}(K, Y)$, which we will eventually prove to be equivalent to the Taylor polynomials of $Q \operatorname{Map}_{*}(K, Y)$. We need some preliminary definitions.

Definition 3. Let $M$ be the category whose objects are the standard finite sets (and the empty set):

$$
\underline{m}=\{1,2,3 \ldots, m\}
$$

and whose morphisms are surjective maps of sets

$$
q: \underline{m^{\prime}} \rightarrow \underline{m}
$$

Let $M_{n}$ be the full subcategory of $M$ whose objects are sets of cardinality $\leq n$.

By abuse of notation, we may sometimes neglect to underline cardinalities and use symbols such as $m$ to denote both the number $m$ and the set $\underline{m}$.

Definition 4. For a finite set $T, X^{\wedge T}$ stands for the total cofiber of the cubical diagram $U \mapsto \operatorname{Map}_{*}\left(U_{+}, X\right), U \subseteq T$, in which the maps $\operatorname{Map}_{*}\left(V_{+}, X\right) \rightarrow$ $\operatorname{Map}_{*}\left(U_{+}, X\right)$ are induced by collapsing maps $U_{+} \rightarrow V_{+}$which send all the elements in $U \backslash V$ to the basepoint.

Basically, $X^{\wedge T}$ is just the smash product of $|T|$ copies of $X$, made functorial in $T$. Note that there is a little twist in case $T=\emptyset$. Our convention is that $X^{\emptyset} \cong *$ and $X^{\wedge \emptyset} \cong *_{+} \cong S^{0}$.

A morphism $q: \underline{m^{\prime}} \rightarrow \underline{m}$ in $M$ induces a well-defined map

$$
\begin{aligned}
& q_{K}: \quad K^{\wedge m} \quad \rightarrow K^{\wedge m^{\prime}}, \\
& k_{1} \wedge k_{2} \wedge \ldots \wedge k_{m} \mapsto k_{q(1)} \wedge k_{q(2)} \wedge \ldots \wedge k_{q\left(m^{\prime}\right)} .
\end{aligned}
$$

In fact, the corrsepondence $\underline{m} \mapsto K^{\wedge m}$ defines a contravariant functor $M \rightarrow T_{*}$.

Definition 5. Let $F_{n}, G_{n}: M_{n} \rightarrow$ Spectra be the two functors defined by

$$
\begin{gathered}
F_{n}(\underline{m})=\Sigma^{\infty} K^{\wedge m}, \\
G_{n}(\underline{m})=\Sigma^{\infty}(Y / X)^{\wedge m} \wedge F(K, X)_{+} .
\end{gathered}
$$


Let $\operatorname{Nat}\left(F_{n}, G_{n}\right)$ be the set of natural transformations from $F_{n}$ to $G_{n}$. Obviously, $\operatorname{Nat}\left(F_{n}, G_{n}\right)$ is a subset of

$$
\prod_{\underline{m} \in \operatorname{Ob}\left(M_{n}\right)} \operatorname{Map}_{*}\left(F_{n}(\underline{m}), G_{n}(\underline{m})\right),
$$

which is the same as

$$
\prod_{m=0}^{n} \operatorname{Map}_{*}\left(K^{\wedge m}, Q\left((Y / X)^{\wedge m} \wedge \operatorname{Map}(K, X)_{+}\right)\right),
$$

and we endow $\operatorname{Nat}\left(F_{n}, G_{n}\right)$ with the subspace topology. Next we need to define fiberwise natural transformations.

Definition 6. The space $\overline{\operatorname{Nat}\left(F_{n}, G_{n}\right)}$ of fiberwise natural transformations is defined to be the (strict) pullback of the diagram

$$
\begin{gathered}
\operatorname{Nat}\left(F_{n}, G_{n}\right) \\
\downarrow \\
\prod_{m=0}^{n} \operatorname{Map}_{*}\left(K^{\wedge m}, Q\left((Y / X)^{\wedge m} \wedge \operatorname{Map}(K, X)_{+}\right)\right) \\
\prod_{m=0}^{n} \frac{\uparrow}{\operatorname{Map}_{*}\left(K^{\wedge m}, Q\left((Y / X)^{\wedge m} \wedge \operatorname{Map}_{*}(K, X)_{+}\right)\right)}
\end{gathered}
$$

where $\overline{\operatorname{Map}_{*}\left(K^{\wedge m}, Q\left((Y / X)^{\wedge m} \wedge \operatorname{Map}_{*}(K, X)_{+}\right)\right)}$is as in definition 2 .

Definition 7. For $n \geq 0$ define

$$
P_{X}^{n} Q \operatorname{Map}_{*}(K, Y)=\overline{\operatorname{Nat}\left(F_{n}, G_{n}\right)} .
$$

We now present an inductive description of $P_{X}^{n} Q \operatorname{Map}_{*}(K, Y)$. Let

$$
\Delta^{n} K=\left\{\left(k_{1} \wedge \ldots \wedge k_{n}\right) \in K^{\wedge n} \mid \exists i, j i \neq j \text { such that } k_{i}=k_{j}\right\} .
$$

Lemma 2. For all $n>0$ there exists a commutative square diagram

$$
\begin{array}{ccc}
P_{X}^{n} \operatorname{QMap}_{*}(K, Y) & \rightarrow & \overline{\operatorname{Map}_{*}\left(K^{\wedge n}, Q\left((Y / X)^{\wedge n} \wedge \operatorname{Map}_{*}(K, X)_{+}\right)\right)^{\Sigma_{n}}} \\
P_{X}^{n-1} \operatorname{QMap}_{*}(K, Y) & \stackrel{t_{n}}{\rightarrow} \frac{\downarrow \tilde{i}}{\operatorname{Map}_{*}\left(\Delta^{n} K, Q\left((Y / X)^{\wedge n} \wedge \operatorname{Map}_{*}(K, X)_{+}\right)\right)^{\Sigma_{n}}}
\end{array}
$$

which is both a strict pullback and a homotopy pullback.

Proof. Following [3], we define the twisted arrow category of $M_{n}$, denoted $a M_{n}$, as follows: The objects of $a M_{n}$ are the morphisms of $M_{n}$, and a morphism $\left(m_{1} \rightarrow\right.$ $\left.m_{2}\right) \rightarrow\left(m_{1}^{\prime} \rightarrow m_{2}^{\prime}\right)$ is a square diagram

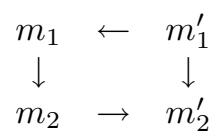

(note the twist in the directions of the horizontal maps). Define the $a M_{n}$-diagram $\operatorname{hom}_{a}\left(F_{n}, G_{n}\right)$ by

$$
\begin{aligned}
\left(m_{1} \rightarrow m_{2}\right) & \mapsto \operatorname{Map}_{*}\left(F_{n}\left(m_{2}\right), G_{n}\left(m_{1}\right)\right) \\
& =\operatorname{Map}_{*}\left(K^{\wedge m_{2}}, Q\left((Y / X)^{\wedge m_{1}} \wedge F(K, X)_{+}\right)\right) .
\end{aligned}
$$


As noted in [3, proposition 3.2], it is easy to see that

$$
\operatorname{Nat}\left(F_{n}, G_{n}\right)=\lim _{\leftarrow}^{a M_{n}} \operatorname{hom}_{a}\left(F_{n}, G_{n}\right) .
$$

We need a fiberwise version of this. Define the diagram $\overline{\operatorname{hom}_{a}\left(F_{n}, G_{n}\right)}$ by

$$
\left(m_{1} \rightarrow m_{2}\right) \mapsto \overline{\operatorname{Map}_{*}\left(K^{\wedge m_{2}}, Q\left((Y / X)^{\wedge m_{1}} \wedge F(K, X)_{+}\right)\right)}
$$

where the right hand side is the image of $\overline{\operatorname{Map}_{*}\left(K^{\wedge m_{2}}, Q\left((Y / X)^{\wedge m_{2}} \wedge F(K, X)_{+}\right)\right)}$ in $\operatorname{Map}_{*}\left(K^{\wedge m_{2}}, Q\left((Y / X)^{\wedge m_{1}} \wedge F(K, X)_{+}\right)\right)$under the inclusion map associated with the surjection $m_{1} \rightarrow m_{2}$. Again it is easy to see that

$$
\overline{\operatorname{Nat}\left(F_{n}, G_{n}\right)}=\lim _{\leftarrow} a M_{n} \overline{\operatorname{hom}_{a}\left(F_{n}, G_{n}\right)} .
$$

Now we write $a M_{n}$ as a union of two categories $a M_{n}^{1}$ and $a M_{n}^{2}$ as follows: Let $a M_{n}^{1}$ be the full subcategory of $a M_{n}$ whose objects are morphisms $m_{1} \rightarrow m_{2}$ such that $m_{2} \leq n-1$. Let $a M_{n}^{2}$ be the full subcategory of $a M_{n}$ whose objects are morphisms $m_{1} \rightarrow m_{2}$ such that $m_{1}=n$. It is easy to see that the nerve of $a M_{n}$ is the union of the nerves of $a M_{n}^{1}$ and $a M_{n}^{2}$, and therefore there is a pullback square

$$
\begin{array}{ll}
\lim ^{a M_{n}} \frac{\operatorname{hom}_{a}\left(F_{n}, G_{n}\right)}{\lim ^{a M_{n}^{2}}} & \rightarrow \quad \underset{\operatorname{hom}_{a}\left(F_{n}, G_{n}\right)}{\lim ^{a M_{n}^{1}} \frac{\downarrow}{\operatorname{hom}_{a}\left(F_{n}, G_{n}\right)}} \rightarrow \underset{\leftarrow}{\lim ^{a M_{n}^{1} \cap a M_{n}^{2}} \frac{\downarrow}{\operatorname{hom}_{a}\left(F_{n}, G_{n}\right)}}
\end{array}
$$

To analyze

$$
\lim _{\leftarrow}^{a M_{n}^{1}} \overline{\operatorname{hom}_{a}\left(F_{n}, G_{n}\right)}
$$

consider the full subcategory of $a M_{n}^{1}$ whose objects are surjections $m_{1} \rightarrow m_{2}$ such that $m_{1} \leq n-1$. It is easy to see that this subcategory is initial in $a M_{n}^{1}$, and therefore

$$
\lim _{\leftarrow}^{a M_{n}^{1}} \overline{\operatorname{hom}_{a}\left(F_{n}, G_{n}\right)} \cong P_{X}^{n-1} Q \operatorname{Map}_{*}(K, Y)
$$

To analyze

$$
\lim _{\leftarrow}^{a M_{n}^{2}} \overline{\operatorname{hom}_{a}\left(F_{n}, G_{n}\right)}
$$

notice that the full subcategory of $a M_{n}^{2}$ whose objects are surjections $n \rightarrow n$ is initial, and therefore

$$
\lim _{\leftarrow}^{a M_{n}^{2}} \overline{\operatorname{hom}_{a}\left(F_{n}, G_{n}\right)} \cong \overline{\operatorname{Map}_{*}\left(K^{\wedge n}, Q\left((Y / X)^{\wedge n} \wedge \operatorname{Map}_{*}(K, X)_{+}\right)\right)^{\Sigma_{n}}} .
$$

Similarly, it is not very hard to check directly that

$$
\lim _{\leftarrow} a M_{n}^{1} \cap a M_{n}^{2} \overline{\operatorname{hom}_{a}\left(F_{n}, G_{n}\right)} \cong \overline{\operatorname{Map}_{*}\left(\Delta^{n} K, Q\left((Y / X)^{\wedge n} \wedge \operatorname{Map}_{*}(K, X)_{+}\right)\right)^{\Sigma_{n}}} .
$$

Thus a pullback square as in the statement of the lemma exists. It is easy to check that the right vertical map is the restriction and thus a fibration by the fibration lemma of section 2. Therefore the square is a homotopy pullback.

Corollary 2. The functor $P_{X}^{n} Q \operatorname{Map}_{*}(K, Y)$ is an excisive functor of degree $n$. Moreover,

$$
D_{X}^{i} P_{X}^{n} Q \operatorname{Map}_{*}(K, Y) \simeq \overline{\operatorname{Map}_{*}\left(K^{(i)}, Q\left((Y / X)^{\wedge i} \wedge \operatorname{Map}_{*}(K, X)_{+}\right)\right)^{\Sigma_{i}}}
$$

for all $0 \leq i \leq n$. 
Proof. It follows from the previous lemma and the corollary of the fibration lemma that the fiber of the map

$$
P_{X}^{n} Q \operatorname{Map}_{*}(K, Y) \rightarrow P_{X}^{n-1} Q \operatorname{Map}_{*}(K, Y)
$$

is homotopy equivalent to

$$
\overline{\operatorname{Map}_{*}\left(K^{(n)}, Q\left((Y / X)^{\wedge n} \wedge \operatorname{Map}_{*}(K, X)_{+}\right)\right)^{\Sigma_{n}},}
$$

which by theorem 1 is a homogeneous functor of degree $n$. The statement follows by induction on $n$.

Next we need to construct natural maps

$$
p_{n}: Q \operatorname{Map}_{*}(K, Y) \rightarrow P_{X}^{n} Q \operatorname{Map}_{*}(K, Y)
$$

such that for all $n$ the diagrams

$$
\begin{array}{ccc}
\operatorname{Map}_{*}(K, Y) & \stackrel{p_{n}}{\rightarrow} & P_{X}^{n} \operatorname{QMap}_{*}(K, Y) \\
& \searrow & \downarrow \\
& & P_{X}^{n-1} \\
& & \operatorname{Map}_{*}(K, Y)
\end{array}
$$

commute, and show that these maps are highly enough connected.

To define $p_{n}$, it is enough to define an unstable map

$$
\widehat{p_{n}}: \operatorname{Map}_{*}(K, Y) \rightarrow \overline{\operatorname{Nat}\left(K^{\wedge n},(Y / X)^{\wedge n} \wedge \operatorname{Map}_{*}(K, X)_{+}\right)} .
$$

We define $\widehat{p_{n}}$ as follows:

$$
\widehat{p_{n}}(f)\left(k_{1}, \ldots, k_{m}\right)=f\left(k_{1}\right) \wedge \ldots \wedge f\left(k_{n}\right) \wedge \bar{f}
$$

where $\bar{f}$ is the composition

$$
K \stackrel{f}{\rightarrow} Y \stackrel{\alpha}{\rightarrow} X .
$$

We are now ready to state our main theorem, whose proof is given in the next subsection.

Theorem 2. The map $p_{n}$ defined above is $(n+1)(k-d)$-connected, where $k$ is the connectivity of $Y / X$ and $d$ is the dimension of $K$.

In other words, $P_{X}^{n} Q \operatorname{Map}_{*}(K, Y)$ is a model for the $n$-th Taylor approximation of $Q \operatorname{Map}_{*}(K, Y)$, and $p_{n}$ is a model for the approximation map.

Remark 2. The reader is encouraged to consider what our formula for

$$
P_{X}^{n} Q \operatorname{Map}_{*}(K, Y)
$$

simplifies to in the special case $X=*$, when "fiberwise" stops being an issue. It might be illuminating to consider the even more special case $K=S^{N}, Y=S^{N} Z$. Letting $N$ go to infinity, one gets formulas for the Taylor tower of $Q Q Z$ (over *), which has to coincide, up to homotopy, with the classical splitting of $Q Q Z$. It is easy to see, for instance, that for $n=2$ our formula says that

$$
P_{*}^{2} Q Q Z \simeq Q_{\Sigma_{2}}\left(Z^{\wedge 2}\right)^{\Sigma_{2}},
$$

which by the tom Dieck splitting is equivalent to $Q(Z) \times Q\left(Z_{h \Sigma_{2}}^{\wedge 2}\right)$ (see [2]), just as one would expect from the Snaith splitting. 
4.2. Proof of the main theorem. It is shown in [5, example 4.5] that the functor $Q\left(\operatorname{Map}_{*}(K, Y)\right)$ is "analytic" enough. Therefore, to show that the map $p_{n}$ is as connected as claimed, it is enough to show that it induces an equivalence on $n$-th Taylor polynomials. For this it is enough to show that $p_{n}$ induces an equivalence on differentials up to the $n$-th one. In fact, by induction it is enough to show that $p_{n}$ induces an equivalence on the $n$-th differential. For this, it is enough to show that the induced map on $n$-th cross-effects, which we denote $\chi_{n} p_{n}$, satisfies the following connectivity condition: if $Z_{1}, \ldots, Z_{n}$ are $k$-connected then $\chi_{n} p_{n}$ is $(n+1) k+c$-connected, where $c$ is a constant not depending on $Z_{1}, \ldots, Z_{n}$.

Let $\left(x_{1}, \ldots, x_{n}\right)$ be an $n$-tuple of points in $X$. It is easy to see from the definitions (see the appendix) that the cross-effect at $\left(x_{1}, \ldots, x_{n}\right)$ satisfies

$$
\begin{aligned}
\left.\chi_{n} Q \operatorname{Map}_{*}(K, Y)\right)\left(Z_{1}, Z_{2}, \ldots, Z_{n}\right) \\
\simeq Q\left(\frac{\operatorname{Map}_{*}\left(K, X \vee_{x_{1}} Z_{1} \vee_{x_{2}} Z_{2} \vee \ldots \vee Z_{n}\right)}{\bigcup_{i=1}^{n} \operatorname{Map}_{*}\left(K, X \vee \ldots \vee \hat{Z}_{i} \vee \ldots\right)}\right)
\end{aligned}
$$

It is also easy to prove, using the fibration lemma and induction on the dimension of $K_{1}^{n}$ in our usual way, that the cross-effect of the functor

$$
\overline{\operatorname{Map}_{*}\left(K_{1}^{n}, Q\left((Y / X)^{n} \wedge \operatorname{Map}_{*}(K, X)_{+}\right)\right)^{\Sigma_{n}}}
$$

satisfies

$$
\begin{aligned}
\chi_{n} & \operatorname{Map}_{*}\left(K_{1}^{n}, Q\left((Y / X)^{\wedge n} \wedge \operatorname{Map}_{*}(K, X)_{+}\right)\right)^{\Sigma_{n}} \\
& \simeq \overline{\operatorname{Map}_{*}\left(K_{1}^{n}, Q\left(\left(\bigvee_{\sigma \in \Sigma_{n}} Z_{\sigma(1)} \wedge \ldots \wedge Z_{\sigma(n)}\right) \wedge \operatorname{Map}_{*}(K, X)_{+}\right)\right)^{\Sigma_{n}}} \\
& \simeq \frac{\operatorname{Map}_{*}\left(K_{1}^{n}, Q\left(\left(Z_{\sigma(1)} \wedge \ldots \wedge Z_{\sigma(n)}\right) \wedge \operatorname{Map}_{*}(K, X)_{+}\right)\right)}{}
\end{aligned}
$$

Moreover, since

$$
\overline{\operatorname{Map}_{*}\left(K^{(n)}, Q\left(Z^{\wedge n} \wedge \operatorname{Map}_{*}(K, X)_{+}\right)\right)^{\Sigma_{n}}}
$$

is the $n$-th differential of $P_{X}^{n} Q \operatorname{Map}_{*}(K, Y)$, the natural map

$$
\overline{\operatorname{Map}_{*}\left(K^{(n)}, Q\left(Z^{\wedge n} \wedge \operatorname{Map}_{*}(K, X)_{+}\right)\right)^{\Sigma_{n}}} \rightarrow P_{X}^{n} Q \operatorname{Map}_{*}(K, Y)
$$

induces an equivalence after applying $\chi_{n}$. Therefore we may regard

$$
\overline{\operatorname{Map}_{*}\left(K^{(n)}, Q\left(\left(\bigvee_{\sigma \in \Sigma_{n}} Z_{\sigma(1)} \wedge \ldots \wedge Z_{\sigma(n)}\right) \wedge \operatorname{Map}_{*}(K, X)_{+}\right)\right)^{\Sigma_{n}}}
$$

as a model for

$$
\chi_{n} P_{X}^{n} Q \operatorname{Map}_{*}(K, Y) .
$$

So, we need to describe $\chi_{n} p_{n}$ in terms of these models for the cross-effects. We are going to describe a map $\chi_{n} \widetilde{p_{n}}$ from

$$
\frac{\operatorname{Map}_{*}\left(K, X \vee Z_{1} \vee Z_{2} \vee \ldots \vee Z_{n}\right)}{\bigcup_{i=1}^{n} \operatorname{Map}_{*}\left(K, X \vee \ldots \vee \hat{Z}_{i} \vee \ldots\right)}
$$


to

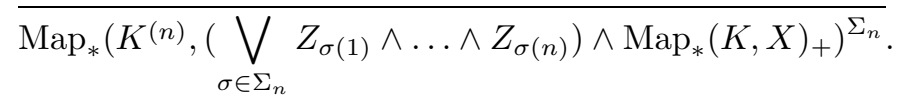

The map is defined as follows: Given a function $f \in \operatorname{Map}_{*}\left(K, X \vee Z_{1} \vee \ldots \vee Z_{n}\right)$, let $\bar{f}$ be the composition

$$
K \stackrel{f}{\rightarrow} X \vee Z_{1} \vee \ldots \vee Z_{n} \rightarrow X
$$

If the point $\left(k_{1}^{1}, k_{1}^{2}, \ldots, k_{1}^{n}\right)$ is different from the basepoint and there exists a permutation $\sigma \in \Sigma_{n}$ such that $f\left(k_{1}^{i}\right) \in Z_{\sigma(i)}$, then

$$
\chi_{n} \widetilde{p_{n}}(f)\left(k_{1}^{1}, k_{1}^{2}, \ldots, k_{1}^{n}\right)=f\left(k_{1}^{\sigma(1)}\right) \wedge f\left(k_{1}^{\sigma(2)}\right) \wedge \ldots \wedge f\left(k_{1}^{\sigma(n)}\right) \wedge \bar{f}
$$

otherwise,

$$
\chi_{n} \widetilde{p_{n}}(f)\left(k_{1}^{1}, k_{1}^{2}, \ldots, k_{1}^{n}\right)=* .
$$

The map $\chi_{n} p_{n}$ is induced by $\chi_{n} \widetilde{p_{n}}$. Our next step is to show that to prove that $\chi_{n} p_{n}$ satisfies the connectivity condition, it is enough to prove that the map $\chi_{n} \widetilde{p_{n}}$ satisfies the connectivity condition.

Proposition 6. Let $Z_{1}, Z_{2}, \ldots, Z_{n}$ be $k$-connected spaces, and let $K_{1}^{n}$ be a subquotient of the space $K^{\times n}$ such that the action of $\Sigma_{n}$ on $K_{1}^{n}$ is free off the basepoint. Then the stabilization map from

$$
\overline{\operatorname{Map}_{*}\left(K_{1}^{n}, \bigvee_{i=1}^{n !}\left(Z_{1} \wedge \ldots \wedge Z_{n}\right) \wedge \operatorname{Map}_{*}(K, X)_{+}\right)^{\Sigma_{n}}}
$$

to

$$
\overline{\operatorname{Map}_{*}\left(K_{1}^{n}, Q\left(\bigvee_{i=1}^{n !}\left(Z_{1} \wedge \ldots \wedge Z_{n}\right) \wedge \operatorname{Map}_{*}(K, X)_{+}\right)\right)^{\Sigma_{n}}}
$$

is $2 n k+c$-connected (and therefore $(n+1) k+c$-connected).

Proof. The idea is the same as usual. That is, to use the fibration lemma to reduce to the case when $K_{1}^{n}$ is an orbit of one point in $K^{\times n}$, in which the map is the inclusion

$$
\begin{gathered}
Z_{1} \wedge \ldots \wedge Z_{n} \wedge \operatorname{Map}_{*}\left(K, k_{1}, \ldots, k_{n} ; X, x_{1} \ldots x_{n}\right)_{+} \\
\downarrow \\
Q\left(Z_{1} \wedge \ldots \wedge Z_{n} \wedge \operatorname{Map}_{*}\left(K, k_{1}, \ldots, k_{n} ; X, x_{1} \ldots x_{n}\right)_{+}\right)
\end{gathered}
$$

which is obviously $2 n k$-connected. As one proceeds with the induction, the connectivity reduces by 1 at each step, so in the end $c=-\operatorname{dim}(K)$.

On the other hand, it is easy to see that if $K$ is a finite complex of dimension $d$, then the space

$$
\frac{\operatorname{Map}_{*}\left(K, X \vee Z_{1} \vee Z_{2} \vee \ldots \vee Z_{n}\right)}{\bigcup_{i=1}^{n} \operatorname{Map}_{*}\left(K, X \vee \ldots \vee \hat{Z}_{i} \vee \ldots\right)}
$$


is $\sim n k-n d$-connected, and therefore the inclusion

$$
\frac{\operatorname{Map}_{*}\left(K, X \vee Z_{1} \vee Z_{2} \vee \ldots \vee Z_{n}\right)}{\bigcup_{i=1}^{n} \operatorname{Map}_{*}\left(K, X \vee \ldots \vee \hat{Z}_{i} \vee \ldots\right)} \hookrightarrow Q\left(\frac{\operatorname{Map}_{*}\left(K, X \vee Z_{1} \vee Z_{2} \vee \ldots \vee Z_{n}\right)}{\bigcup_{i=1}^{n} \operatorname{Map}_{*}\left(K, X \vee \ldots \vee \hat{Z}_{i} \vee \ldots\right)}\right)
$$

is $2 n k-2 n d$-connected, and in particular is $\sim(n+1) k+c$-connected.

Therefore, it is enough to prove that the map $\chi_{n} \widetilde{p_{n}}$ has the required connectivity property.

It is enough to prove it in the case when $K=M$ is a parallelizable compact $m$-dimensional Riemannian manifold with boundary and a basepoint $m_{0}$. It also follows from Goodwillie's classification of homogeneous functors that we may assume $Z_{i} \cong S^{m} W_{i}$. In this case we can use the machinery of configuration spaces. We will need suitable fiberwise versions of the classical results on approximation of mapping spaces by configuration spaces.

Remark 3. The proof would have been much easier if we were content to prove the case $X \cong *$, which already is of interest. In this case we would only need classical results from the theory of configuration spaces.

Let $M$ be as above. Let $C(M ; j)=\left(M \backslash\left\{m_{0}\right\}\right)^{\times j} \backslash \Delta^{j} M$ be the space of $j$-tuples of distinct points in $M \backslash\left\{m_{0}\right\}$. Let $C_{\epsilon}(M ; j)$ be the space of $j$-tuples of points in $M \backslash\left\{m_{0}\right\}$ such that the distance between any two is at least $3 \epsilon$ and so is the distance from the basepoint. Let $x \in X$.

\section{Definition 8.}

$$
\begin{aligned}
\overline{C(M ; j) \times_{x} \operatorname{Map}_{*}(M, X)}= & \left\{\left(m_{1}, m_{2}, \ldots m_{j}, f\right) \in C(M ; j) \times \operatorname{Map}_{*}(M, X) \mid\right. \\
& \left.f\left(m_{i}\right)=x \forall 1 \leq i \leq j\right\} .
\end{aligned}
$$

For $W$ a based space, make the following definition:

\section{Definition 9.}

$$
\overline{C_{X, x ; W}(M)}=\coprod_{j \geq 0}\left(\overline{C(M ; j) \times_{x} \operatorname{Map}_{*}(M, X)} \times W^{j}\right)_{\approx},
$$

where $\approx$ stands for the usual identifications:

(i)

$$
\begin{array}{ccc}
\left(\left\langle m_{1}, \ldots, m_{i}, m_{i+2}, \ldots, m_{j}\right\rangle,\right. & f & \left.,\left\langle w_{1}, \ldots, w_{i}, w_{i+2}, \ldots, w_{j}\right\rangle\right) \\
\approx\left(\left\langle m_{1}, \ldots, m_{i}, m_{i+1}, m_{i+2}, \ldots, m_{j}\right\rangle,\right. & f & \left.,\left\langle w_{1}, \ldots, w_{i}, *, w_{i+2}, \ldots, w_{j}\right\rangle\right)
\end{array}
$$

(ii)

$$
\begin{array}{ccc}
\left(\left\langle m_{1}, \ldots, m_{j}\right\rangle,\right. & f & \left.,\left\langle w_{1}, \ldots, w_{j}\right\rangle\right) \\
\approx\left(\left\langle m_{\sigma(1)}, \ldots, m_{\sigma}(j)\right\rangle,\right. & f & \left.,\left\langle w_{\sigma(1)}, \ldots, w_{\sigma(j)}\right\rangle\right)
\end{array}
$$

$$
\begin{array}{ccc}
\left(\left\langle m_{1}, \ldots, m_{i}, \ldots, m_{j}\right\rangle,\right. & f & \left.,\left\langle w_{1}, \ldots, w_{i}, \ldots, w_{j}\right\rangle\right) \\
\approx\left(\left\langle m_{1}, \ldots, \hat{m}_{i}, \ldots, m_{j}\right\rangle,\right. & f & \left.,\left\langle w_{1}, \ldots, \hat{w}_{i}, \ldots, w_{j}\right\rangle\right)
\end{array}
$$

if $m_{i} \in \partial M$. 
Similarly define the space $\overline{C_{\epsilon ; X, x ; W}(M)}$. There is a map

$$
\bigcup_{\epsilon \geq 0} \overline{C_{\epsilon ; X, x ; W}(M)} \times\{\epsilon\} \rightarrow \overline{C_{X, x ; W}(M)}
$$

which is a homotopy equivalence.

Let $N$ and $N^{\prime}$ be two manifolds of the same dimension as $M$. Suppose that they are embedded as closed submanifolds of $M$ in such a way that $M=N \cup N^{\prime}$ and $N \cap N^{\prime}=\partial N \cap \partial N^{\prime}$.

Definition 10. We say that the inclusion $N \hookrightarrow M$ is nice if

1) $B=\partial N \cap \partial N^{\prime}$ is a submanifold of $\partial N$ and of $\partial N^{\prime}$, and

2) each connected component of $B$ has nonempty intersection with $\partial M$.

We will need the following:

Lemma 3. Let $N \hookrightarrow M$ be a nice inclusion. Then the restriction map

$$
\begin{gathered}
\overline{C_{X, x ; W}(M)} \\
\frac{\downarrow r}{C_{X, x ; W}(N)}
\end{gathered}
$$

is a quasifibration.

Proof. The proof is an adaptation of the corresponding proof in [9]. Filter the space

$$
\coprod_{j \geq 0}\left(\overline{C(N ; j) \times_{x} \operatorname{Map}_{*}(N, X)} \times W^{j}\right)_{\approx}
$$

by the spaces

$$
\overline{C_{k}}=\coprod_{j=0}^{j=k}\left(\overline{C(N ; j) \times{ }_{x} \operatorname{Map}_{*}(N, X)} \times W^{j}\right) \approx .
$$

To prove that the map $r$ is a quasifibration it is enough to prove that:

1) $r: r^{-1}\left(\overline{C_{k+1}} \backslash \overline{C_{k}}\right) \rightarrow \overline{C_{k+1}} \backslash \overline{C_{k}}$ is a fibration with the same fiber for all $k$.

2) For each $k$ there is an open subset $U_{k}$ of $\overline{C_{k}}$ which contains $\overline{C_{k-1}}$ and there are homotopies

$$
h_{t}: U_{k} \rightarrow U_{k}
$$

and

$$
H_{t}: r^{-1}\left(U_{k}\right) \rightarrow r^{-1}\left(U_{k}\right)
$$

such that

a) $h_{0}=\mathrm{id}, h_{t}\left(\overline{C_{k-1}}\right) \subseteq \overline{C_{k-1}}, h_{1}\left(U_{k}\right) \subseteq \overline{C_{k-1}}$;

b) $H_{0}=\mathrm{id}, r H_{t}=h_{t} r$;

c) $H_{1}: r^{-1}(x) \rightarrow r^{-1}\left(h_{1}(x)\right)$ is a homotopy equivalence for all $x \in U_{k}$.

To prove (1) we need to show that for any commutative square of the form

$$
\begin{array}{cccc}
I^{n} & \stackrel{G}{\rightarrow} & r^{-1} & \left(\left(\overline{C(N ; j) \times_{x} \operatorname{Map}_{*}(N, X)} \times\left(W^{\wedge j} \backslash *\right)\right)_{\Sigma_{j}}\right) \\
\downarrow & & \downarrow r \\
I^{n+1} & \stackrel{F}{\rightarrow} & \left(\overline{C(N ; j) \times_{x} \operatorname{Map}_{*}(N, X)} \times\left(W^{\wedge j} \backslash *\right)\right)_{\Sigma_{j}}
\end{array}
$$


there is a map

$$
E: I^{n+1} \rightarrow r^{-1}\left(\left(\overline{C(N ; j) \times_{x} \operatorname{Map}_{*}(N, X)} \times\left(W^{\wedge j} \backslash *\right)\right)_{\Sigma_{j}}\right)
$$

which commutes with all other maps in the square.

To prove this we introduce some more notation. Since the action of all symmetric groups on $\operatorname{Map}_{*}(M, X)$ is trivial, it is clear that

$$
\coprod_{j \geq 0}\left(\overline{C(M ; j) \times \operatorname{Map}_{*}(M, X)} \times W^{j}\right)_{\approx}
$$

is homeomorphic to

$$
\overline{\left(\coprod_{j \geq 0}\left(C(M ; j) \times W^{j}\right)\right)_{\approx} \times \operatorname{Map}_{*}(M, X)}
$$

So we will think of the functions $F, G$, and $E$ as products $F=F_{1} \times F_{2}, G=G_{1} \times G_{2}$, $E=E_{1} \times E_{2}$, where $F_{1}$ maps $I^{n+1}$ to $\overline{\left(C(N ; j) \times\left(W^{\wedge j} \backslash *\right)\right)_{\Sigma_{j}}}, F_{2}$ maps $I^{n+1}$ to $\operatorname{Map}_{*}(N, X)$, and so on. Of course the pairs $\left(F_{1}, F_{2}\right),\left(G_{1}, G_{2}\right),\left(E_{1}, E_{2}\right)$ must satisfy an additional condition, a version of the bar condition.

We denote $I^{n+1}=\left\{(u, t) \in I^{n} \times I\right\}$. Also, we think of a point in

$$
\left(\coprod_{j \geq 0} C(M ; j) \times W^{j}\right)_{\approx}
$$

as a finite subset $[S]$ of $M \backslash \partial M$ whose points are labeled by points in $W$ which are different from the basepoint. Then, given two points $[S]$ and $[U]$, one can obviously define standard operations of set theory on them, such as their union $[S \cup U]$, their set difference $[S \backslash U]$, etc.

We will construct the map

$$
E: I^{n+1} \rightarrow r^{-1}\left(\left(\overline{C(N ; j) \times_{x} \operatorname{Map}_{*}(N, X)} \times\left(W^{\wedge j}\right) \backslash *\right)_{\Sigma_{n}}\right)
$$

by constructing maps

$$
E_{1}: I^{n+1} \rightarrow\left(\coprod_{j \geq 0} C(M ; j) \times W^{j}\right)_{\approx}
$$

and

$$
E_{2}: I^{n+1} \rightarrow \operatorname{Map}_{*}(M, X)
$$

in such a way that they satisfy the bar condition and the image of $E=E_{1} \times E_{2}$ is in $r^{-1}\left(\left(\overline{C(N ; j) \times_{x} \operatorname{Map}_{*}(N, X)} \times\left(W^{\wedge j} \backslash *\right)\right)_{\Sigma_{n}}\right)$ and the map $E$ commutes with other maps in the square diagram.

First we define

$$
E_{1}((u, t))=\left[F_{1}(u, t) \cup\left(G_{1}(u) \backslash N\right)\right]
$$


Clearly, this map is well defined and continuous. To show that it is possible to define a map $E_{2}$ such that $E_{1} \times E_{2}$ has all the desired properties we need to show that there is a map

$$
E_{2}: I^{n+1} \rightarrow \operatorname{Map}_{*}(M, X)
$$

such that:

1) the composition $I^{n+1} \stackrel{E_{2}}{\rightarrow} \operatorname{Map}_{*}(M, X) \stackrel{\text { restriction }}{\rightarrow} \operatorname{Map}_{*}(N, X)$ is equal to $F_{2}$;

2) the composition $I^{n} \rightarrow I^{n+1} \stackrel{E_{2}}{\rightarrow} \operatorname{Map}_{*}(M, X)$ is equal to $G_{2}$.

3) $E_{2}((u, t))(m)=x \forall(u, t) \in I^{n+1} \forall m \in\left(\operatorname{Im}\left(E_{1}(u, t)\right) \backslash N\right)$.

So we can describe the situation as follows: we need to define a map

$$
\widetilde{E_{2}}: I^{\times n} \times I \times M \rightarrow X
$$

which is already predetermined on the subset

$$
I^{n} \times M \cup I^{n+1} \times N \cup I^{n+1} \times\left(\operatorname{Im}\left(G_{1}\right) \backslash N\right) .
$$

Moreover, the restriction of the map to $I^{n+1} \times\left(\operatorname{Im}\left(G_{1}\right) \backslash N\right)$ is a constant map. Clearly, the subspace $\left(\operatorname{Im}\left(G_{1}\right) \backslash N\right)$ is separated from $N$ in $M$, and there exists a closed subspace $O \subset M$ such that:

(i) $\left(\operatorname{Im}\left(G_{1}\right) \backslash N\right) \subset O$,

(ii) $O$ has an open neighborhood disjoint with $N$,

(iii) $(M, N \cup O)$ is an NDR pair.

Choose $O$ as above and extend $\widetilde{E_{2}}$ to $I^{n} \times M \cup I^{n+1} \times N \cup I^{n+1} \times O$ in the obvious way (sending $O$ to the point $x$ ). Now the map can be extended to all of $I^{n+1} \times M$, since $(M, N \cup O)$ is an NDR pair. This completes the proof of condition (1) in our lemma.

By our assumption on $B$ we may choose $\epsilon>0$ so that the set $\{y \in M: d(y, B)<$ $2 \epsilon\}$ is homeomorphic to to $B \times(-2 \epsilon, 2 \epsilon)$. Also, for small $\epsilon>0, N \backslash N_{2 \epsilon}$ is homeomorphic to $\partial N \times[0,2 \epsilon)$. It follows that there is a homotopy $l_{t}:(M, \partial M) \rightarrow(M, \partial M)$ such that $l_{0}=$ id, $l_{1}\left(N_{\epsilon}\right)=N$ and $l_{t} \mid l_{t}^{-1}(M \backslash \partial M)$ is injective. Because of this injectivity of $l_{t}$, it induces a homotopy

$$
\left(l_{t}\right)_{*}: \overline{C_{X, W}(M)} \rightarrow \overline{C_{X, W}(M)} .
$$

Let $U_{k}$ be $\left\{[S] \in \overline{C_{k}(N)} \mid \operatorname{card}\left(S \cap N_{\epsilon}\right) \leq k-1\right\}$ and let $h_{t}, H_{t}$ be the appropriate restrictions of $\left(l_{t}\right)_{*}$. Clearly, subconditions (a) and (b) of condition (2) are satisfied. It remains to prove that

$$
H_{1}: r^{-1}([S], f) \rightarrow r^{-1}\left(h_{1}[S], f\right)
$$

is a homotopy equivalence for all $([S], f)$ in $U_{k}$

Clearly, each fiber $r^{-1}([S], f)$ is canonically isomorphic to

$$
V=\coprod_{j \geq 0}\left(\overline{C\left(N^{\prime}, \overline{\partial N^{\prime} \backslash B}\right) \times \operatorname{Map}_{*}\left(N^{\prime}, B ; X, f(B)\right)} \times W^{j}\right)_{\approx} ;
$$

in terms of this identification $H_{1}$ is a map $H_{1}: V \rightarrow V$. In fact it is

$$
\left([U],\left.f\right|_{N^{\prime}}\right) \mapsto\left(\left[l_{1}(U) \cup w\right],\left.f \circ l_{1}\right|_{N^{\prime}}\right),
$$

where $[w]=\left[l_{1}(S) \cap N^{\prime}\right]$ is a configuration in the neighborhood $W=l_{1}(N) \cap N^{\prime}$ of $B$ in $N^{\prime}$. Now $\left[l_{1}(U)\right]$ lies outside $W$ for all $[U] \in\left(C\left(N^{\prime}, \overline{\partial N^{\prime} \backslash B}\right) \times W^{j}\right){ }_{\approx}$. Also, we assumed that each component of $B$ has nonempty intersection with $\partial \widetilde{\widetilde{M}}$, 
so that the configuration $[w]$ can be connected to the empty configurations through configurations in $W$. Therefore the map

$$
\left([U],\left.f\right|_{N^{\prime}}\right) \mapsto\left(\left[l_{1}(U) \cup w\right], f \circ l_{1} \mid N^{\prime}\right)
$$

is homotopic to

$$
\left([U],\left.f\right|_{N^{\prime}}\right) \mapsto\left(\left[l_{1}(U)\right],\left.f \circ l_{1} \circ(\text { a homotopy equivalence of } M)\right|_{N^{\prime}}\right)
$$

and this is homotopic to $\operatorname{id}_{F}$ since $l_{1} \simeq$ id.

We are now ready to prove the following fact.

Theorem 3. Let $M$ be a parallelizable manifold of dimension $m$ with nonempty boundary. There is a map

$$
\coprod_{j \geq}\left(\overline{C_{\epsilon}(M ; j) \times_{x} \operatorname{Map}_{*}(M, X)} \times W^{j}\right)_{\approx} \rightarrow \operatorname{Map}_{*}\left(M, X \vee_{x} S^{m} W\right)
$$

which is a homotopy equivalence.

Proof. The map is defined the usual way. For each point $m_{i}$, labeled by a point $w_{i}$, map the $\epsilon$-ball centered at $m_{i}$ to the sphere over $w_{i}$ in $S^{m} W$ (since $M$ is parallelizable, we can do this in a canonical way) and map the rest of $M$ to $X$ as determined by the map $f \in \operatorname{Map}_{*}(M, X)$, using the fact that the space obtained from $M$ by identifying $j \epsilon$-balls to their centers is homeomorphic to $M$. To prove that this map is an equivalence we need only to check that the assertion is true for the case

$$
M=S^{0} \times D^{m}
$$

Then the assertion for general $M$ follows by induction on handles, which works thanks to the previous lemma. See [9] for details.

In the case $M=S^{0} \times D^{m}$ it is clear that

$$
\operatorname{Map}_{*}\left(M, X \vee S^{m} W\right) \cong \operatorname{Map}_{*}\left(D^{m}, X \vee S^{m} W\right) \times \operatorname{Map}_{*}\left(D_{+}^{m}, X \vee S^{m} W\right)
$$

and

$$
\begin{aligned}
& \coprod_{j \geq 0}\left(\overline{C(M ; j) \times_{x} \operatorname{Map}_{*}(M, X)} \times W^{j}\right)_{\approx} \\
& \cong \coprod_{j \geq 0}\left(\overline{C\left(D^{m} ; j\right) \times_{x} \operatorname{Map}_{*}\left(D^{m}, X\right)} \times W^{j}\right)_{\approx} \\
& \quad \times \coprod_{j \geq 0}\left(\overline{C\left(D^{m} ; j\right) \times_{x} \operatorname{Map}_{*}\left(D_{+}^{m}, X\right)} \times W^{j}\right)_{\approx} .
\end{aligned}
$$

So, we need to prove that the maps

$$
\coprod_{j \geq 0}\left(\overline{C\left(D^{m} ; j\right) \times_{x} \operatorname{Map}_{*}\left(D^{m}, X\right)} \times W^{j}\right)_{\approx} \rightarrow \operatorname{Map}_{*}\left(D^{m}, X \vee S^{m} W\right)
$$

and

$$
\coprod_{j \geq 0}\left(\overline{C\left(D^{m} ; j\right) \times_{x} \operatorname{Map}_{*}\left(D_{+}^{m}, X\right)} \times W^{j}\right) \approx \operatorname{Map}_{*}\left(D_{+}^{m}, X \vee S^{m} W\right)
$$

are equivalences. This is easy to do directly and is left as an exercise to the reader. 
As a consequence, we get a generalization of the classical "stable splitting" theorem.

Lemma 4. Let $M$ be an $m$-manifold. Then there are stable equivalences

$$
\begin{aligned}
\operatorname{Map}_{*}\left(M, X \vee_{x} S^{m} W\right) \simeq \overline{C_{X, x, W}(M)} \\
\quad \rightarrow \bigvee_{j=0}^{j=\infty} \overline{C(M, \partial M ; j) \wedge_{x} \operatorname{Map}_{*}(M, X)_{+}} \wedge_{\Sigma_{j}} W^{\wedge j}
\end{aligned}
$$

Proof. We have just proved the first equivalence in theorem 3. As for the second equivalence, its meaning is that the filtration of the "fiberwise configuration space" model stably splits into the wedge sum of its subquotients, which are easily seen to be

$$
\overline{C(M, \partial M ; j) \wedge_{x} \operatorname{Map}_{*}(M, X)_{+}} \wedge W^{\wedge j} .
$$

The construction and proof of the second equivalence carry over from the proof of the corresponding statement in [1]

Corollary 3. Let $M$ be a parallelizable, compact, m-dimensional manifold with boundary. The $n$-th differential of the functor $\operatorname{Map}_{*}\left(M, X \vee_{x} S^{m} W\right)$ is given by

$$
D_{X}^{n} Q \operatorname{Map}_{*}\left(M, X \vee_{x} S^{m} W\right) \simeq Q\left(\overline{C(M, \partial M ; n) \wedge_{x} \operatorname{Map}_{*}(M, X)_{+}} \wedge W^{\wedge n}\right)_{\Sigma_{n}} .
$$

Proof. By theorem 3. There is an equivalence

$$
\coprod_{j \geq 0}\left(\overline{C_{\epsilon}(M ; j) \times_{x} \operatorname{Map}_{*}(M, X)} \times W^{j}\right) \approx \operatorname{Map}_{*}\left(M, X \vee S^{m} W\right),
$$

where the space on the right is filtered by the spaces

$$
\overline{C_{n}}=\coprod_{j=0}^{j=n}\left(\overline{C_{\epsilon}(M ; j) \times{ }_{x} \operatorname{Map}_{*}(M, X)} \times W^{j}\right)_{\approx},
$$

and it is easy to see that

$$
\overline{C_{n}} / \overline{C_{n-1}} \cong\left(\overline{C(M, \partial M ; n) \wedge_{x} \operatorname{Map}_{*}(M, X)_{+}} \wedge W^{\wedge n}\right)_{\Sigma_{n}} .
$$

Clearly, if $W$ is $l$-connected, then the quotient $\overline{C_{n}} / \overline{C_{n-1}}$ is $n l$-connected. In particular, this implies that the inclusion

$$
\overline{C_{n}} \hookrightarrow \coprod_{j \geq 0}\left(\overline{C(M ; j) \times_{x} \operatorname{Map}_{*}(M, X)} \times W^{j}\right)_{\approx}
$$

is $(n+1) l$-connected. On the other hand, by lemma 4

$$
\coprod_{j \geq 0}\left(\overline{C(M ; j) \times_{x} \operatorname{Map}_{*}(M, X)} \times W^{j}\right)_{\approx}
$$

stably splits into the product of the quotients. To summarize, there is an equivalence

$$
Q \operatorname{Map}_{*}\left(M, X \vee S^{m} W\right) \simeq \prod_{j \geq 0} Q\left(\left(\overline{C(M, \partial M ; j) \wedge_{x} \operatorname{Map}_{*}(M, X)_{+}} \wedge W^{\wedge j}\right)_{\Sigma_{j}}\right) .
$$


Clearly, $Q\left(\left(\overline{C(M, \partial M ; j) \wedge_{x} \operatorname{Map}_{*}(M, X)_{+}} \wedge W^{\wedge j}\right)_{\Sigma_{j}}\right)$ is a homogeneous functor of degree $j$, and therefore the functor

$$
\prod_{j=0}^{j=n} Q\left(\left(\overline{C(M, \partial M ; j) \wedge_{x} \operatorname{Map}_{*}(M, X)_{+}} \wedge W^{\wedge j}\right)_{\Sigma_{j}}\right)
$$

is a functor of degree $n$. Since there is a (weak) map

$$
\operatorname{Map}_{*}\left(M, X \vee S^{m} W\right) \rightarrow \prod_{j=0}^{j=n} Q\left(\left(\overline{C(M, \partial M ; j) \wedge_{x} \operatorname{Map}_{*}(M, X)_{+}} \wedge W^{\wedge j}\right)_{\Sigma_{j}}\right)
$$

which is $(n+1) l$-connected whenever $W$ is $l$-connected, it must be true, by uniqueness and universality of Goodwillie's "Taylor approximations", that

$$
P_{n} Q \operatorname{Map}_{*}\left(M, X \vee S^{m} W\right) \simeq \prod_{j=0}^{j=n} Q\left(\left(\overline{C(M, \partial M ; j) \wedge_{x} \operatorname{Map}_{*}(M, X)_{+}} \wedge W^{\wedge j}\right)_{\Sigma_{j}}\right)
$$

and

$$
D_{n} Q \operatorname{Map}_{*}\left(M, X \vee S^{m} W\right) \simeq Q\left(\left(\overline{C(M, \partial M ; n) \wedge \operatorname{Map}_{*}(M, X)_{+}} \wedge W^{\wedge n}\right)_{\Sigma_{n}}\right) .
$$

It is clear now that, given an $n$-tuple $\left(x_{1}, \ldots, x_{n}\right)$ of points in $X$, the space of maps

$$
\operatorname{Map}_{*}\left(M, X \vee_{x_{1}} S^{m} W_{1} \vee \ldots \vee_{x_{n}} S^{m} W_{n}\right)
$$

can be approximated by means of appropriate fiberwise configuration spaces. More specifically, it is clear that there is a map from

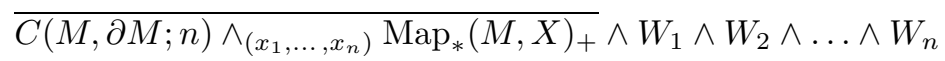

(defined in the obvious way) to

$$
\frac{\operatorname{Map}_{*}\left(M, X \vee S^{m} W_{1} \vee S^{m} W_{2} \vee \ldots \vee S^{m} W_{n}\right)}{\bigcup_{i=1}^{n} \operatorname{Map}_{*}\left(K, X \vee \ldots \vee \widehat{S^{m} W_{i}} \vee \ldots\right)}
$$

which is $(n+1) k$-connected whenever all $W_{i}$ 's are $k$-connected. It remains to prove that the composed map

$$
\begin{gathered}
\overline{C(M, \partial M ; n) \wedge\left(x_{1}, \ldots, x_{n}\right)} \operatorname{Map}_{*}(M, X)_{+} \wedge W_{1} \wedge W_{2} \wedge \ldots \wedge W_{n} \\
\frac{\operatorname{Map}_{*}\left(M, X \vee_{x_{1}} S^{m} W_{1} \vee_{x_{2}} S^{m} W_{2} \vee \ldots \vee_{x_{n}} S^{m} W_{n}\right)}{\bigcup_{i=1}^{n} \operatorname{Map}_{*}\left(K, X \vee \ldots \vee \widehat{S^{m} W_{i}} \vee \ldots\right)} \\
\frac{\downarrow}{\operatorname{Map}_{*}\left(M^{(n)}, S^{m} W_{1} \wedge \ldots \wedge S^{m} W_{n} \wedge \operatorname{Map}_{*}(K, X)\right)}
\end{gathered}
$$

satisfies the same connectivity condition.

In fact, we can prove a more general statement: 
Lemma 5. Let $U=M^{(n)}$ (so $U \backslash *$ is a manifold). Let $U_{1}$ be a subspace of $U$. We will say that the inclusion $U_{1} \hookrightarrow U$ is nice, if $U \backslash *$ is a manifold and the inclusion $U_{1} \backslash * \hookrightarrow U \backslash *$ is nice. Then there is a configuration space model for

$$
\overline{\operatorname{Map}_{*}\left(U_{1},\left(\operatorname{Map}_{*}(M, X)_{+} \wedge S^{m} W_{1} \wedge \ldots \wedge S^{m} W_{n}\right)\right)} \text {. }
$$

More precisely, there is an equivalence

$$
\begin{gathered}
\coprod_{i \geq 0} \overline{\left(C\left(U_{1} \backslash * ; i\right) \times\left(\operatorname{Map}_{*}(M, X)_{+} \wedge\left(W_{1} \wedge W_{2} \wedge \ldots \wedge W_{n}\right)\right)^{i}\right)} \\
\frac{\downarrow}{\operatorname{Map}_{*}\left(U_{1}, Q\left(\operatorname{Map}_{*}(M, X)_{+} \wedge S^{m} W_{1} \wedge \ldots \wedge S^{m} W_{n}\right)\right) .}
\end{gathered}
$$

Proof. The idea of the proof is the same as in lemma 3. Given a subspace $U_{2}$ of $U_{1}$, such that the inclusion $U_{2} \hookrightarrow U_{1}$ is nice, there is the following proposition

Proposition 7. The restriction map

$$
\begin{aligned}
& \coprod_{i \geq 0}\left(\overline{\left(C\left(U_{1} \backslash * ; i\right) \times\left(\operatorname{Map}_{*}(M, X)_{+} \wedge\left(W_{1} \wedge W_{2} \wedge \ldots \wedge W_{n}\right)\right)^{i}\right.}\right) \approx \\
& \coprod_{i \geq 0} \overline{\left(C\left(U_{2} \backslash * ; i\right) \times\left(\operatorname{Map}_{*}(M, X)_{+} \wedge\left(W_{1} \wedge W_{2} \wedge \ldots \wedge W_{n}\right)\right)^{i}\right)}
\end{aligned}
$$

is a quasifibration.

Proof. The proof is similar to that of lemma 3, only easier.

Using this proposition, our lemma is proved by induction on handles.

\section{Appendix A. TAYlor TOWERS}

Following the conventions of $([4],[5],[6])$, we say that a functor from spaces to spaces is a homotopy functor, if it takes weak equivalences to weak equivalences and commutes with mapping telescopes. We will work with homotopy functors $F: T_{X} \rightarrow D$, where $T_{X}$ is the category of spaces containing a fixed space $X$ as a retract and $D$ is either the category of based spaces or the category of spectra. The calculus of functors is concerned with approximating such homotopy functors by functors of a special kind, the so-called excisive functors or functors of finite degree, which should be interpreted as "polynomial functors".

The definition of excisive functors involves cubical diagrams of spaces (see [5] for definitions). A cubical diagram of spaces is said to be strongly co-Cartesian if each of its two-dimensional faces is a homotopy pushout square. A cubical diagram of spaces is said to be Cartesian if it is a homotopy pullback cube. A homotopy functor $F$ is said to have degree $n$ or equivalently to be $n$-excisive if it satisfies $n^{\text {th }}$ order excision. This means that it takes $n+1$ strongly co-Cartesian cubical diagrams to $n+1$ Cartesian cubical diagrams. For instance, a functor is 1-excisive (linear) if it takes homotopy pushout squares to homotopy pullback squares. An example of a linear functor is $Q Y=\Omega^{\infty} \Sigma^{\infty} Y$. In general, any linear functor $T_{*} \rightarrow D$ has (up to natural weak homotopy equivalence) the form $\Omega^{\infty}(\mathbf{E} \wedge Y)$, where $\mathbf{E}$ is some spectrum.

A homotopy functor $F$ is said to be analytic if, loosely speaking, it satisfies $n$-th order excision in a stable range for all $n$ (see [5, definition 4.2] for a precise definition). Many of the familiar homotopy functors are analytic. For instance 
the identity functor, Waldhausen's A-theory, and $\operatorname{Map}(K, X)$, where $K$ is a finite CW-complex, are analytic. All functors dealt with in this paper are analytic.

One of the main results of calculus of functors is that an analytic functor can be approximated by functors of finite degree in much the same way as an analytic function can be approximated by its Taylor polynomials. That is, to any analytic functor there corresponds a tower of functors $\left\{P_{n} F\right\}_{n \geq 0}$ of degree $n$ with maps $\ldots \rightarrow P_{n} F \rightarrow P_{n-1} F \rightarrow \ldots$ This tower is uniquely determined (up to a natural weak equivalence) by the universal property that for each $n$ there is a natural map $F(X) \rightarrow P_{n} F(X)$ that is roughly $(n+1) k$-connected (here $k$ is the connectivity of $Y$ in $T_{X}$ ). This tower of functors is called the Taylor tower of the functor $F$.

A functor $F$ is homogeneous of degree $n$ if it is of degree $n$ and $P_{n-1} F \simeq *$. It is proved in [6] that the fibers of the maps $P_{n} F \rightarrow P_{n-1} F$ are homogeneous functors (of degree $n$ ). These functors are called the differentials of $F$ and are usually denoted $D_{n} F$.

In [6] Goodwillie classified all homogeneous functors (whose domain is $T_{*}$ ) up to natural weak equivalence. A homogeneous functor of degree $n$ is determined by a spectrum $\mathbf{A}$ endowed with an action of the group $\Sigma_{n}$ and it has, up to natural weak equivalence, the form $\Omega^{\infty}\left[\left(\mathbf{A} \wedge Y^{\wedge n}\right)_{h \Sigma_{n}}\right]$. Its visual resemblance to the function $\frac{a x^{n}}{n !}$ further enhances the analogy with Taylor polynomials. Let $F: T_{*} \rightarrow D$ be a functor with $D_{n} F(Y) \simeq \Omega^{\infty}\left(\left(\mathbf{A} \wedge Y^{\wedge n}\right)_{h \Sigma_{n}}\right)$; then we say that the spectrum A, together with the action of $\Sigma_{n}$, is the $n$-th derivative of $F$ at $*$ (of course, the derivative of a functor is determined only up to a suitable notion of weak $\Sigma_{n}$ equivariant pseudo-equivalence). Thus the $n$-th derivative of a functor defined on $T_{*}$ is a spectrum with an action of $\Sigma_{n}$, or equivalently, a bundle of spectra over $B \Sigma_{n}$.

For a general space $X$, the $n$-th derivative of a functor $F: T_{X} \rightarrow D$ is a bundle of spectra over $X^{n} \times_{\Sigma_{n}} E \Sigma_{n}$. Equivalently, it is a bundle of spectra over $X^{n}$ with an action of $\Sigma_{n}$. The best way to define the $n$-th derivative is probably via the $n$-th "cross-effect" of $F$. By definition, the $n$-th cross-effect of a functor $F: T_{X} \rightarrow D$ is a bundle over $X^{n}$ of functors $\chi_{n} F: T_{*}^{\times n} \rightarrow D$. The fiber at an $n$ tuple $\left(x_{1}, \ldots, x_{n}\right)$ of this bundle is the iterated homotopy fiber of the $n$-dimensional cube $S \mapsto F\left(X \vee_{x_{i_{1}}} Z_{i_{1}} \vee \ldots \vee_{x_{i_{k}}} Z_{i_{k}}\right)$, where $\left\{i_{1}, \ldots, i_{k}\right\}$ is the complement of $S$ in $\{1, \ldots, n\}$. The maps in the cube are induced by the obvious collapsing maps. It is easily seen from the definitions that there is an action of $\Sigma_{n}$ on $\chi_{n} F$ and that each fiber is a reduced functor of $\left(Z_{1}, \ldots, Z_{n}\right)$. It follows that the multilinearization of a fiber of $\chi_{n} F$ is given, up to homotopy, by

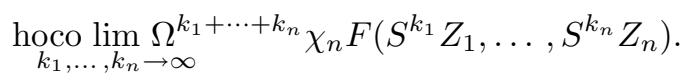

We denote this functor by $D^{(n)} F . D^{(n)} F$ is a symmetric multilinear functor, and as such is represented by a spectrum with an action of $\Sigma_{n}$. This gives a bundle of spectra over $X^{n} \times_{\Sigma_{n}} E \Sigma_{n}$.

Obviously, there is some work in making all this precise, but it is not really difficult.

The following theorem is proved in [6]

Theorem 4. Let $F, G: T_{X} \rightarrow$ Spaces $_{*}$ be two homogeneous functors of degree $n$. Let $h: F \rightarrow G$ be a natural transformation. $h$ is a weak equivalence if and only if it induces a weak equivalence of $n$-th derivatives. 


\section{REFERENCES}

1. C.-F. Bödigheimer, Stable splitting of mapping spaces, Springer Lecture Notes, Vol. 1286 (1987), 174-187. MR 89c:55011

2. G. Carlsson, Survey of equivariant stable homotopy theory, Topology 31 (1992), 1-27. MR 93d:55009

3. W.G. Dwyer and D.M. Kan, Function complexes for diagrams of simplicial sets, Indagationes Mathematicae 45 (1983), 139-147. MR 85e:55038

4. T.G. Goodwillie, Calculus I: the first derivative of pseudoisotopy theory, K-Theory 4 (1990), 1-27. MR 92m:57027

5. T.G. Goodwillie, Calculus II: analytic functors, K-Theory 5 (1992), 295-332. MR 93i:55015

6. T.G. Goodwillie, Calculus III: the Taylor series of a homotopy functor, in preparation.

7. J.P. May, The geometry of iterated loop spaces, Lecture Notes in Mathematics 271 (1972). MR 54:8623b

8. J.P. May, Weak equivalences and quasifibrations, Springer Lecture Notes, Vol. 1425 (1990), 91-101. MR 91m:55016

9. D. McDuff, Configuration spaces of positive and negative particles, Topology 14 (1975), 91107. MR 50:11225

10. G. Segal, Categories and cohomology theories, Topology 13 (1974), 293-312. MR 50:5782

Department of Mathematics, University of Chicago, Chicago, Illinois 60637

E-mail address: arone@math.uchicago.edu 